\title{
Minor mergers and their impact on the kinematics of old and young stellar populations in disk galaxies ${ }^{\star}$
}

\author{
Y. $\mathrm{Qu}^{1}$, P. Di Matteo ${ }^{1}$, M. D. Lehnert ${ }^{1}$, W. van Driel ${ }^{1}$, and C. J. Jog ${ }^{2}$ \\ ${ }^{1}$ GEPI, Observatoire de Paris, CNRS, Université Paris Diderot, 5 place Jules Janssen, 92190 Meudon, France \\ e-mail: yan.qu@obspm.fr \\ 2 Department of Physics, Indian Institute of Science, Bangalore 560012, India
}

Received 12 January 2011 / Accepted 21 September 2011

\begin{abstract}
By means of $N$-body simulations we investigate the impact of minor mergers on the angular momentum and dynamical properties of the merger remnant. Our simulations cover a range of initial orbital characteristics and gas-to-stellar mass fractions (from 0 to $20 \%$ ), and include star formation and supernova feedback. We confirm and extend previous results by showing that the specific angular momentum of the stellar component always decreases independently of the orbital parameters or morphology of the satellite, and that the decrease in the rotation velocity of the primary galaxy is accompanied by a change in the anisotropy of the orbits. However, the decrease affects only the old stellar population, and not the new population formed from gas during the merging process. This means that the merging process induces an increasing difference in the rotational support of the old and young stellar components, with the old one lagging with respect to the new. Even if our models are not intended specifically to reproduce the Milky Way and its accretion history, we find that, under certain conditions, the modeled rotational lag found is compatible with that observed in the Milky Way disk, thus indicating that minor mergers can be a viable way to produce it. The lag can increase with the vertical distance from the disk midplane, but only if the satellite is accreted along a direct orbit, and in all cases the main contribution to the lag comes from stars originally in the primary disk rather than from stars in the satellite galaxy. We also discuss the possibility of creating counter-rotating stars in the remnant disk, their fraction as a function of the vertical distance from the galaxy midplane, and the cumulative effect of multiple mergers on their creation.
\end{abstract}

Key words. galaxies: kinematics and dynamics - galaxies: evolution - galaxies: interactions - galaxies: formation

\section{Introduction}

Rotationally supported disks account for only a small fraction of the mass in the local Universe, but they contain most of the angular momentum (AM). The way disks acquire and redistribute their AM represents one of the most challenging problems for models of galaxy formation and evolution. According to the current cosmological paradigm, baryons and dark halos in galaxies acquire their spin through tidal torques exerted by adjacent structures at early times. This AM is then redistributed among the different galaxy components through a number of internal and external processes as the galaxy evolves. Among the internal processes, bars, lopsidedness, spiral patterns and other coherent structures are efficient in redistributing AM in galaxies, as many studies have shown (e.g., Athanassoula 2005; Debattista et al. 2006; Minchev et al. 2011). These stellar asymmetries can be stimulated or strengthened by external processes, such as accretion of a few $M_{\odot} \mathrm{yr}^{-1}$ of gas from cosmological filaments (see Bournaud et al. 2005a,b) or tidal interactions and mergers (Jog \& Maybhate 2006; Mapelli et al. 2008; Reichard et al. 2009). In particular, during an interaction orbital AM is converted into internal rotation, in an outside-in manner: the components which first interact are the most extended ones, while the more tightly bound components experience strong tidal effects only in the final phases of the merging process (Barnes 1992; Di Matteo et al. 2008b, 2009).

* Figures 9-18 are available in electronic form at http://www . aanda.org
Many studies have shown that major mergers have a catastrophic impact on the ordered motion of the pre-existing galaxies. If the progenitors have disks, they are usually destroyed by the strong energy and AM redistribution taking place during the interaction (Toomre 1977; Bendo \& Barnes 2000; Naab \& Burkert 2003; Bournaud et al. 2005c; Jesseit et al. 2009), unless peculiar orbital configurations are chosen (Puerari \& Pfenniger 2001; Crocker et al. 2009). The fraction of gas present in the progenitor disks can also influence the morphology and kinematics of the final remnant (e.g., Hopkins et al. 2009), but this depends as well on the gas physics implemented in the models (e.g., Bournaud et al. 2011). In the case of pressure-supported progenitors, in turn, the tidal torques exerted by the companion can be strong enough to produce high rotational support $(v / \sigma>1)$ at large radii, even in merger remnants having an elliptical-like morphology (Di Matteo et al. 2009). More attention has been given to the study of the impact of AM redistribution in major mergers than in minor mergers (with mass ratios $\leq 0.1$ ). This despite the fact that minor mergers are expected to be much more common than major mergers (Fakhouri \& Ma 2008) and that many traces of ongoing or past interactions are visible both in the Milky Way (see Klement 2010, for a recent review), our neighbor galaxy Andromeda (Ibata et al. 2001; McConnachie et al. 2009) and other galaxies in the local Universe (Martinez-Delgado et al. 2010). Di Matteo et al. (2010) pointed out that the AM redistribution during minor mergers also have an impact on the kinematics of stellar disks and may explain the distribution of the orbital eccentricities of stars in 
the solar neighborhood. Understanding how AM is redistributed during such episodes is fundamental to the understanding of how disks can be maintained, how their kinematics can be affected, and what the signatures are of these processes on the dynamical properties of different stellar populations.

This paper is the second of a series where we study, by means of numerical simulations, the impact of minor mergers on AM redistribution in galaxies. In Qu et al. (2010) (hereafter Paper I), we investigated the impact of dissipationless minor mergers on disk galaxies, showing in particular that the initially non-rotating dark matter halo of the primary galaxy always gains AM and that the specific AM of the stellar component always decreases. We also showed that this decrease in AM is accompanied by a change in stellar velocity anisotropy as the stellar orbits become less tangentially dominated as the merger advances. In this paper, we aim to advance this analysis by studying simulations of dissipative minor mergers, exploring a range of gas fractions and morphological parameters for the primary galaxy and the satellite. Star formation and feedback from supernovae explosions are included in the models, and we are able to trace the AM redistribution of all the galaxy components: dark matter, gas, old stars (i.e., those already in the galaxies before the interaction starts) and new stars (i.e., those formed from the gas during the interaction). In particular, we aim to understand if dissipative minor mergers still slow down the stellar disk of the primary galaxy and if stellar populations of different ages show a different AM content and different dynamical properties in the final (i.e., postmerger) disk.

The paper is organized as follows: the numerical code, the initial galaxy models and orbital conditions adopted for the runs are described in Sect. 2. Section 3 presents the main results, in particular how the AM content of gas and the old and new stellar populations is affected by a single and by two consecutive (Sect. 3.1) minor mergers, and how the rotational lag induced by this redistribution affects the old stellar component (Sect. 3.2). The contribution of accreted stars to the rotational lag, and the possibility to distinguish them on the basis of their AM content, are discussed in Sect. 3.3. Section 3.4 discusses the effect of single and two consecutive retrograde mergers on the fraction of counter-rotating stars. Finally, Sect. 4 presents a discussion of the results found and the main conclusions.

\section{Models}

All 121 simulations described in this paper are part of the GalMer project ${ }^{1}$ and were fully described in Chilingarian et al. (2010). Here we recall the main characteristics of the adopted galaxy models and orbital parameters, as well as those of the numerical code employed to run the simulations.

In this paper, we study the interaction and successive merger of a satellite galaxy with a ten times more massive primary disk galaxy. Both the primary and the satellite consist of a spherical non-rotating dark halo and a central bulge, both modeled by Plummer spheres of masses $M_{\mathrm{H}}$ and $M_{\mathrm{B}}$, respectively, and core radii $r_{\mathrm{H}}$ and $r_{\mathrm{B}}$, a stellar and an optional gas disk, represented by Myamoto-Nagai density profiles of masses $M_{*}$ and $M_{\text {gas }}$, disk scale lengths $a_{*}$ and $a_{\text {gas }}$, and scale heights $h_{*}$ and $h_{\text {gas }}$, respectively. Both the primary and the satellite galaxies span a range of morphologies, with a variety of bulge to disk ratios, and gasto-stellar mass fractions (from $f_{\text {gas }}=0$ to $f_{\text {gas }}=20 \%$ ). Moving from early to late type systems, we refer to these models respectively with the nomenclature $\mathrm{gS} 0, \mathrm{gSa}$ and $\mathrm{gSb}$ for the primary

\footnotetext{
${ }^{1}$ http://galmer.obspm.fr
}

Table 1. Parameters for the initial models of the bulge, halo and stellar and gaseous disks.

\begin{tabular}{|c|c|c|c|c|c|c|c|}
\hline & gS0 & $\mathrm{gSa}$ & $\mathrm{gSb}$ & $\mathrm{dE} 0$ & dS0 & $\mathrm{dSa}$ & $\mathrm{dSb}$ \\
\hline$M_{\mathrm{B}}\left[2.3 \times 10^{9} M_{\odot}\right]$ & 10. & 10. & 5. & 7. & 1 & 1 & 0.5 \\
\hline$M_{\mathrm{H}}\left[2.3 \times 10^{9} M_{\odot}\right]$ & 50. & 50. & 75. & 3. & 5 & 5 & 7.5 \\
\hline$M_{*}\left[2.3 \times 10^{9} M_{\odot}\right]$ & 40. & 40. & 20. & 0 & 4 & 4 & 2 \\
\hline$M_{\mathrm{gas}} / M_{*}$ & - & 0.1 & 0.2 & - & - & 0.1 & 0.2 \\
\hline$r_{\mathrm{B}}[\mathrm{kpc}]$ & 2. & 2. & 1. & 1.3 & 0.6 & 0.6 & 0.3 \\
\hline$r_{\mathrm{H}}[\mathrm{kpc}]$ & 10. & 10. & 12. & 2.2 & 3.2 & 3.2 & 3.8 \\
\hline$a_{*}[\mathrm{kpc}]$ & 4. & 4. & 5. & - & 1.3 & 1.3 & 1.6 \\
\hline$h_{*}[\mathrm{kpc}]$ & 0.5 & 0.5 & 0.5 & - & 0.16 & 0.16 & 0.16 \\
\hline$a_{\text {gas }}[\mathrm{kpc}]$ & - & 5. & 6. & - & - & 1.6 & 1.9 \\
\hline$h_{\mathrm{gas}}[\mathrm{kpc}]$ & - & 0.2 & 0.2 & - & - & 0.06 & 0.06 \\
\hline
\end{tabular}

Table 2. Particle numbers for primary disk galaxies and satellites.

\begin{tabular}{cccc}
\hline \hline & $\mathrm{gS} 0$ & $\mathrm{gSa}$ & $\mathrm{gSb}$ \\
\hline$N_{\text {gas }}$ & - & 80000 & 160000 \\
$N_{\text {star }}$ & 320000 & 240000 & 160000 \\
$\mathrm{~N}_{\text {DM }}$ & 160000 & 160000 & 160000 \\
\hline
\end{tabular}

\begin{tabular}{ccccc}
\hline \hline & $\mathrm{dE} 0$ & $\mathrm{dS} 0$ & $\mathrm{dSa}$ & $\mathrm{dSb}$ \\
\hline$N_{\text {gas }}$ & - & - & 8000 & 16000 \\
$N_{\text {star }}$ & 32000 & 32000 & 24000 & 16000 \\
$N_{\text {DM }}$ & 16000 & 16000 & 16000 & 16000 \\
\hline
\end{tabular}

galaxy, and dS0, dSa and dSb for the satellite. For the satellites, we also consider a simple spheroid dominated model, consisting only of a dark halo and a stellar bulge, without any disk component - we refer to this model as dE0. All the parameters of the galaxies described above are given in Table 1, and the number of particles adopted to describe the different galaxy components in Table 2.

As we have done in Qu et al. (2010), all galaxy models were first evolved in isolation for $1 \mathrm{Gyr}$ before the interaction starts, to let the initial system reach a stable configuration. Once relaxed, the two galaxies were placed at an initial distance of $100 \mathrm{kpc}$, with a variety of relative velocities, to simulate different orbits (see Chilingarian et al. 2010, Table 9, for the orbital initial conditions). To study the effect of multiple mergers, we also ran some simulations in which the primary galaxy accretes consecutively two or three satellites over a period of 3-5 Gyr. We also compared the dynamical properties of disks after minor mergers to those heated by internal processes, such as scattering by massive clumps formed in an initially unstable disk (Bournaud et al. 2009). For this, we analyzed a simulation of a gas-rich, unstable disk galaxy from Di Matteo et al. (2008a). It uses a total number of 120000 particles, equally distributed among gas, stars and dark matter, and has the same parameters as the gSb model (see Table 1) except for the gas mass fraction, which is initially much higher, $50 \%$.

All the simulations were run using the Tree-SPH code described in Semelin \& Combes (2002). Gas is treated as isothermal, at a fixed temperature of $T=10^{4} \mathrm{~K}$. Prescriptions for star formation and feedback from supernovae explosions are also included. The rate of star formation is governed locally by a volume density Schmidt law $\dot{M}_{\text {gas }} \propto \rho^{\alpha} M_{\text {gas }}$ with $\alpha=1.5$, which is implemented in the code by means of a hybrid particle approach (see Mihos \& Hernquist 1994; Chilingarian et al. 2010 for details). At each time step, a hybrid particle is characterized by two mass values: the first is its total mass $M_{i}$ which stays constant 
Y. Qu et al.: Minor mergers and their impact on the kinematics of old and young stellar populations in disk galaxies

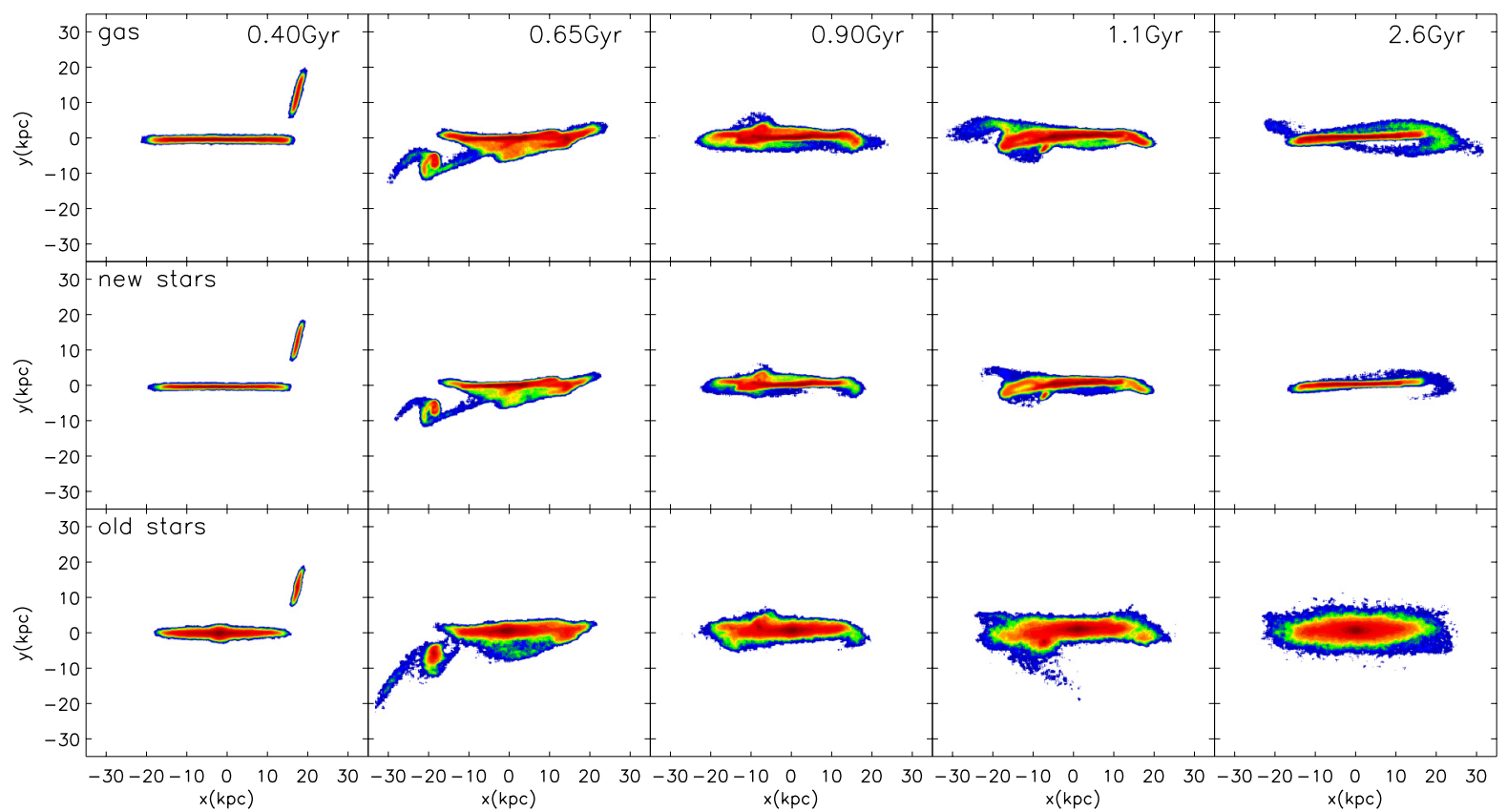

Fig. 1. From left to right: evolution with time of gas (upper panels) and stars (middle and lower panels) during a minor merger between a gSb and a dSb galaxy on a direct orbit (gSbdSb01dir33). The stellar component is separated into new stars (formed during the interaction, middle row of panels), and old stars (those already present in the galaxies before the interaction, lower panels). In this projection the disk of the primary galaxy is viewed edge-on.

during the simulation and is used to calculate the gravitational force, and the second is given by the gas content of the particle, $M_{\mathrm{i}, \mathrm{gas}}$, which changes with time according to the Schmidt law and is used to evaluate hydrodynamical quantities. At each time step the mass of the new stars, i.e. those formed since the beginning of the simulation, is given by $M_{\mathrm{i}}-M_{\mathrm{gas}}$. If $M_{\mathrm{i}, \mathrm{gas}}$ drops below $5 \%$ of the initial value, the "hybrid" particle is considered to be totally converted into a star-like particle and its small residual amount of gas spread out over its (hybrid) neighbors. Note that in the hybrid particle scheme, new stars follow the gas kinematics until the moment when the hybrid particle they reside in is completely converted into stars. This can represent a limitation of our approach, in the sense that it does not allow us to properly follow the heating with time of the newly formed stars by secular processes. Finally, for the evaluation of the gravitational forces a softening length $\epsilon=200 \mathrm{pc}$ is employed. The equations of motion are integrated using a leap-frog algorithm, with a fixed time step of $0.5 \mathrm{Myr}$. With these choices, the relative error in the conservation of the total energy is close to $10^{-6}$ per time step.

The nomenclature adopted for our simulations consists of a string of 13 characters: the first three (gS0, gSa or gSb) for the primary galaxy and the following three for the satellite galaxy (dE0, dS0, dSa or dSb), followed by the encounter identification string (see Chilingarian et al. 2010, Table 9) and the orientation of the disk of the primary galaxy with respect to the orbital plane (33 or 60 degrees). Multiple mergers are indicated by two more characters $-\mathrm{tn}$, where $\mathrm{n}$ is the number of satellites accreted by the primary galaxy. For example, the string gSadSat202dir33 refers to a gSa galaxy accreting two dwarf dSa galaxies, whose initial orbital parameters are those corresponding to the string 02dir in Table 9 of Chilingarian et al. (2010).

\section{Results}

In Paper I, we showed that during dissipationless minor mergers the orbital $\mathrm{AM}$ is redistributed into internal $\mathrm{AM}$ of the interacting galaxies in an outside-in manner: the initially nonrotating dark halos of primary and satellite start to acquire AM first, just after the first pericenter passage between the two galaxies, while the strongest changes in the primary stellar disk take place mostly in the final phase of the collision, when the satellite is close to merging. All our simulations also showed that the stellar disk loses part of its initial AM. The aim of this section is to investigate if these results are also valid for dissipative mergers, if differences can be found in the rotational support of the different stellar populations in the primary disk once the merger is completed and what their main kinematic characteristics are.

\subsection{Gas, old stars, new stars and the evolution of their angular momenta}

As discussed in Sect. 2, our model galaxies contain both "old stars", which were already present before the start of the interaction, and "new stars" formed from gas during the interaction. We will follow the nomenclature of old and new stars throughout the text. An example of the spatial distribution and morphological evolution of these two stellar components, as well as the dissipative gas component, is shown in Fig. 1. Before the first close passage of the satellite, which occurs at $0.5 \mathrm{Gyr}$, gas and new stars are distributed in a morphologically thin and dynamically cold disk, and old stars in a thicker and hotter component and in a central bulge. At $0.65 \mathrm{Gyr}$, the primary disk shows signs of a tidal perturbation: some gas and new stars are clearly displaced from the midplane of the primary, and the old stellar disk starts to thicken even further. Note also that the satellite morphology has already been strongly modified at this time. Signs of tidal perturbation of the primary galaxy are visible all along the merging sequence after the first close passage of the satellite. At 2.6 Gyr, or more than $1 \mathrm{Gyr}$ after the merger ended, its morphology has clearly been profoundly modified: a thick disk made of old stars has been formed (see Qu et al. 2011, for a description of its 
morphological properties) whereas gas and new stars are still distributed in a thin disk component with some perturbations at large radii, such as a warp and a tidal tail.

During the interaction, the effects of dynamical friction and tidal forces redistribute the AM in the system: both the primary and the satellite galaxy acquire part of the orbital AM, and, as already found in Paper I, the most extended regions experience these changes first. In particular, the impact of a minor merger on the AM content of the primary galaxy is shown in Fig. 2, where the evolution with time of the specific AM of old stars, gas, new stars and dark matter is shown for different regions of the galaxy. The figure shows clearly that:

- the outermost regions of the initially non-rotating dark halo are the first to acquire part of the orbital AM, already after the first pericenter passage, at $t=0.5 \mathrm{Gyr}$, while inside $3 R_{\mathrm{d}}$ (the initial disk scale length $R_{\mathrm{d}}$ is $4.8,4.8$ and $5.2 \mathrm{kpc}$ for S0, $\mathrm{Sa}$ and $\mathrm{Sb}$ type galaxies, respectively) tend to increase their AM only during the final phases of the merger;

- the AM of the baryonic components is also affected by the interaction. The old stars experience a slowing down of their rotation at all radii, especially outside $0.5 R_{\mathrm{d}}$. A less pronounced decrease of the AM is visible in the gas (and new stars) between $R_{\mathrm{d}}$ and $3 R_{\mathrm{d}}$. For example, in the merger case shown in Fig. 2 the fractional decrease in the specific AM of old stars is $\Delta l / l=0.19$ at $2-3 R_{\mathrm{d}}$ whereas $\Delta l / l=0.09$ for gas (and new stars) in the same region. While the outermost regions gain AM in the innermost ones the specific AM remains unchanged.

The slowing down with time of the AM content of old stars at a given radius is not only due to disk heating, but also an effect of the merger process (as has been previously shown by Quillen et al. 2009). To demonstrate this effect, in Fig. 3 we show the total AM of old stars, gas and new stars (for dissipative mergers), and dark matter of the primary galaxy for three simulations with different gas fractions in the primary disk $(0,0.1$ and 0.2$)$. In all cases, one can see a substantial decrease of the total AM of old stars $(\Delta L / L=0.13$ for a $\mathrm{gSbdSb}$ direct merger, 0.2 for a gSadSa merger and 0.36 for a dissipationless merger). Also the gas (and new stars) tend to lose a fraction of their AM. At the same time, the dark halo of the primary galaxy acquires part of the AM, which is already apparent after the first passage of the satellite, around $t=0.5 \mathrm{Gyr}$ in the simulations. These substantial changes in AM content of the different components are clearly an effect of the minor merger itself. This can be seen when comparing the evolution of the merging galaxies with that of the corresponding galaxies which were evolved in isolation (right panels of Fig. 3). Secular processes, which dominate the evolution of the isolated galaxy simulations, cause less pronounced changes in the AM, with the decrease in $\Delta L / L$ of the old stellar disk being only about $20-25 \%$ of what is estimated for the minor merger simulations. This demonstrates that disk heating due to secular processes, which increases the radial stellar velocity dispersions, cannot be the only mechanism responsible for the slowing down of the disks observed in the minor merger simulations.

Figures 2 and 3 suggest that it should be possible to find a difference in the AM content of old and new stars in the final, post-merger disk, as is indeed the case, see Fig. 4: comparing the specific AM as a function of radius for gas and new stars (left panel) and old stars (middle panel), one can see that while the AM of the new stellar component is unchanged after the merger, that of the old stars decreases at all radii $\left(\Delta l / l=0.23\right.$ at $\left.R_{\mathrm{d}}\right)$. This is due to the fact that, during the merging process, old stars
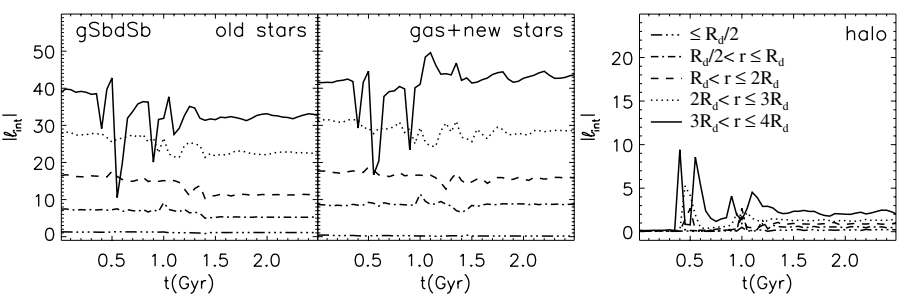

Fig. 2. Evolution with time of the specific AM, $l$, of a gSb primary galaxy during the minor merger whose evolution is shown in Fig. 1. The specific AM of old stars (left panel), gas and new stars (middle panel) and dark matter (right panel) is shown, for five different radial zones in the galaxy, see the legend. $R_{\mathrm{d}}$ indicates the disk scale length. The specific AM is in units of $100 \mathrm{kpc} \mathrm{km} \mathrm{s}^{-1}$.

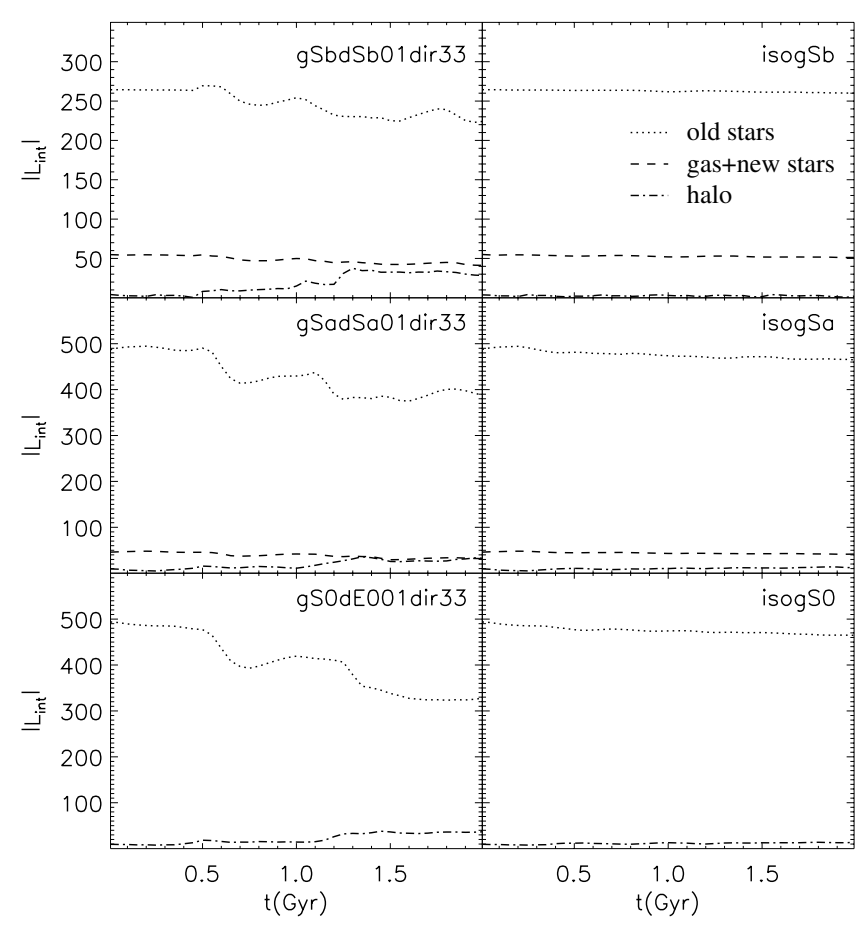

Fig. 3. Left panels: evolution with time of the total AM of gas + new stars (dashed line), old stars (dotted line) and the dark matter halo (dashed-dotted line) in the region at $r<20 \mathrm{kpc}$ from the center of the primary galaxy during a minor merger with a satellite galaxy on a direct orbit. From top to bottom, the gas mass fraction of both primary galaxy and merging satellite are $20 \%, 10 \%$ and 0 , respectively. The AM is in units of $2.2 \times 10^{11} M_{\odot} \mathrm{kpc} \mathrm{km} \mathrm{s}^{-1}$. Right panels: the evolution of the AM of the corresponding components (gas + new stars, old stars and dark matter halo) of isolated galaxies with the same initial structure as the primary galaxies in the minor merger simulations.

are heated as shown by the increasing radial and vertical velocity dispersion (Fig. 5), which then leads to a slowing down of the stellar disk, as we have argued in Paper I. However, gas can dissipate its energy, and thus can preserve mainly tangential motion and keep its AM unaffected, whereas stars newly formed from this gas retain the AM that was acquired by the gas. This means that at a given radius the old and the new stellar populations have a different specific AM and thus different amounts of rotational support. As discussed in Paper I for dissipationless mergers, the decrease in the rotation speed of the old stellar disk of the primary galaxy is accompanied by a change in the distribution of the types of stellar orbits: the radial component of the 
Y. Qu et al.: Minor mergers and their impact on the kinematics of old and young stellar populations in disk galaxies
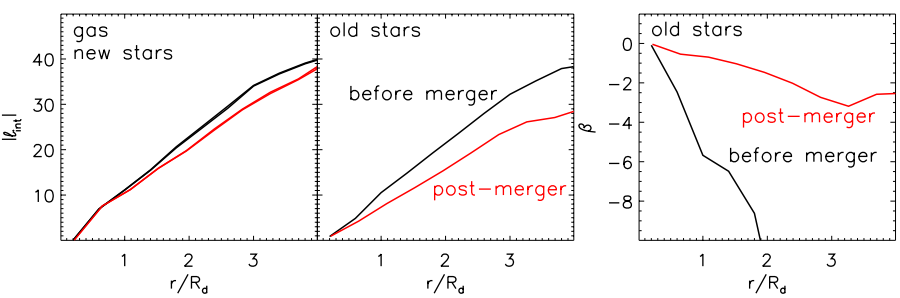

Fig. 4. From left to right: specific AM as a function of radius normalized by the disk scale length, $r / R_{\mathrm{d}}$, of the primary galaxy, for gas and new stars (left panel) and old stars (middle panel) during the minor merger simulation whose evolution is shown in Fig. 1. In each panel the black line shows the initial AM and the red line shows the final AM. The specific AM is in units of $100 \mathrm{kpc} \mathrm{km} \mathrm{s}^{-1}$. Right panel: the anisotropy parameter $\beta$ as a function of $r / R_{\mathrm{d}}$ for old stars in the same merger. Both the initial (black line) and final (red line) $\beta$ are shown.
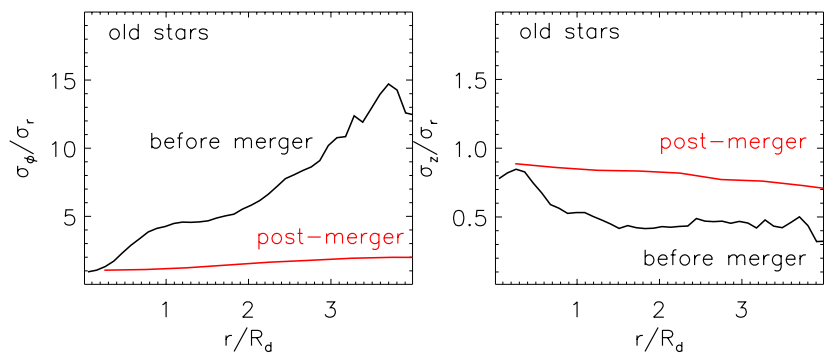

Fig. 5. Left panel: ratio of tangential and radial velocity dispersion as a function of scaled radius $r / R_{\mathrm{d}}$ for old stars before (black line) and after (red line) the minor merger shown in Fig.1. Right panel: same as the left panel but for the ratio of vertical and radial velocity dispersion.

velocity dispersion becomes increasingly important during the merger, thus increasing the anisotropy parameter, $\beta=1-\frac{\sigma_{\mathrm{t}}^{2}}{2 \sigma_{\mathrm{r}}^{2}}$, from its initially negative value. This is also the case for dissipative minor mergers (Fig. 4). Independent of the amount of gas present in the primary disk or in the satellite galaxy, minor mergers always result in a slowing down of the old stellar disk. The fractional decrease in the specific AM at $R_{\mathrm{d}}$ is $\Delta l / l=0.23$ for a gSb model, $\Delta l / l=0.17$ for a $\mathrm{gSa}$ and $\Delta l / l=0.29$ for a $\mathrm{gS} 0$.

Note that the slowing down of the old stellar disk during the merger is also visible in the time evolution of the AM of those stars that were initially (at $t=0$ ) present in a given radial bin, rather than in that of stars currently present at a given radius (Fig. 6). At any given initial radius, $r_{\text {ini }}$, the variation of the AM is much stronger when the stellar disk undergoes a minor merger than when it evolves in isolation. In the minor merger simulations, the average value of $\Delta l / l$ decreases with time, especially for stars whose initial radii exceed $R_{\mathrm{d}}$. The largest changes take place in the outer disk where the effect of the perturbations due to the satellite is the greatest (as already has been shown by Quillen et al. 2009; Bird et al. 2011).

\subsubsection{The impact of multiple mergers}

If a single minor merger causes the slowing down of the old stellar component of a galaxy disk, what would be the effect of a subsequent minor merger on it? To answer this question, we ran ten simulations in which the primary galaxy consecutively accretes two ten times less massive satellites over a period of 3-5 Gyr. The second minor merger produces a further decrease in the specific AM of the old stellar population (Fig. 7). After the first merger, the specific AM decreases at all radii, accompanied by an increase of the $\beta$ parameter. In the second merger event,
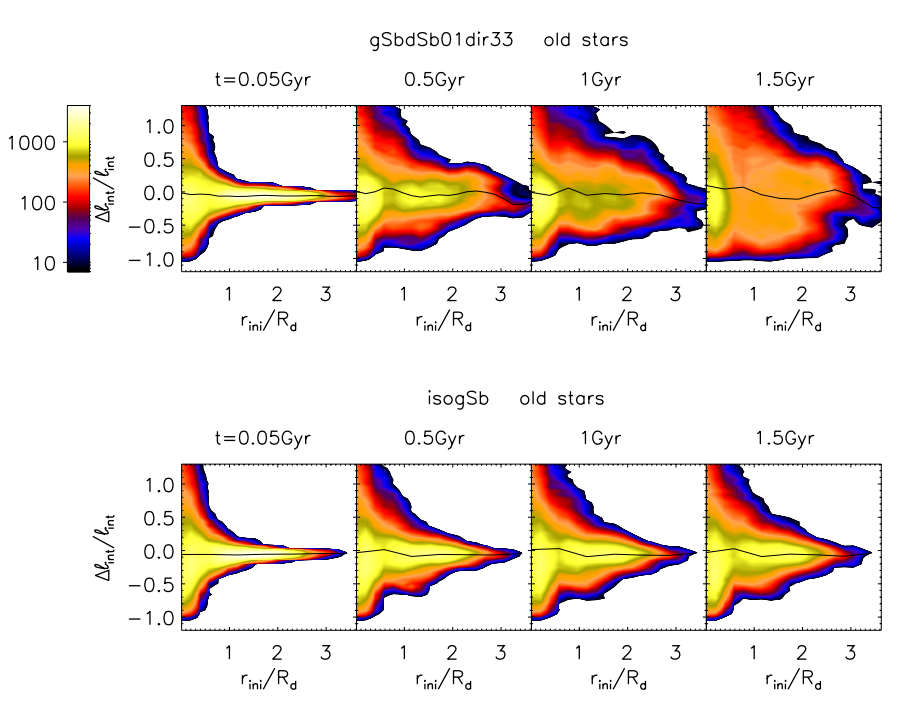

Fig. 6. Upper panels: relative change of the specific AM with respect to the initial value, $\Delta l_{\text {int }} / l_{\text {int }}=\left(l_{\text {int }}(t)-l_{\text {int }}(t=0)\right) / l_{\text {int }}(t=0)$, of old stars of a gSb galaxy as a function of initial radius $\mathrm{r}_{\text {ini }}$ (scaled by $R_{\mathrm{d}}$ ) during a minor merger with a dSb galaxy on a direct orbit (gSbdSb01dir33). Also shown is the average of $\Delta l_{\text {int }} / l_{\text {int }}$ as a function of $r_{\text {ini }} / R_{\mathrm{d}}$ in each epoch (overlaid solid line). The vertical bar on the left shows the color scale indicating the number of old stars at certain $\Delta l_{\text {int }} / l_{\text {int }}$ values. Lower panels: the distribution of $\Delta l_{\text {int }} / l_{\text {int }}$ of old stars of an isolated galaxy with the same initial structure as the primary galaxy in the minor merger case.
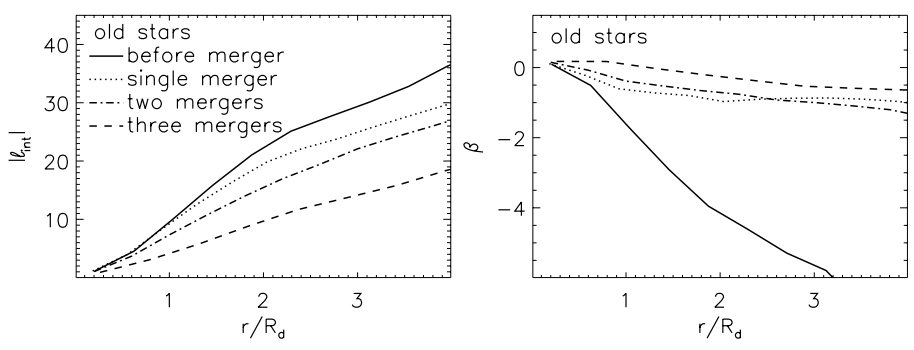

Fig. 7. Left panel: specific $\mathrm{AM}$ as a function of scaled radius $r / R_{\mathrm{d}}$ for the old stars of a gSa galaxy undergoing three consecutive minor mergers. The specific AM is shown after the first (dotted line), the second (dashed-dotted line), and the third (dashed line) merger, and the initial AM is shown for comparison (solid line). The specific AM is in units of $100 \mathrm{kpc} \mathrm{km} \mathrm{s}^{-1}$. Right panel: anisotropy parameter, $\beta$, as a function of scaled radius, for the old stars of the gSa galaxy whose specific AM is shown in the left panel.

the AM content of the old disk stellar component is further decreased and this is accompanied by an increase in the velocity anisotropy parameter $\beta$ at all radii in the disk. Furthermore, we ran one simulation in which the primary galaxy consecutively accretes three ten times less massive satellites over a period of 5 Gyr. After having accreted two satellites the stellar disk of the primary galaxy still reacted dynamically to the third satellite accretion - its specific AM decreased further, its beta parameter increased and the slowing down of the old stellar disk did not saturate but increased (see Fig. 7). While old stars slow down with time, whereas stars formed during the repeated mergers tend to show little change in their AM (Fig. 8), the difference in AM content between stars born at different times increases during repeated mergers with low mass satellite galaxies. This is only partially due to the hybrid method used to implement star formation in our simulations, in which the new stars are 

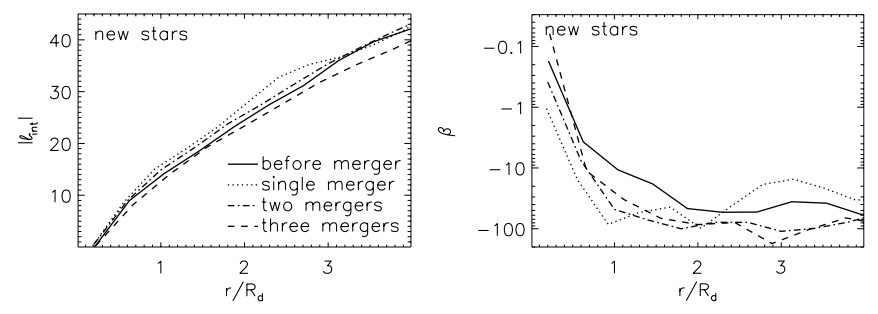

Fig. 8. Same as Fig. 7 but for newly formed stars in the gSa galaxy.

continuously formed from dissipative gas which keeps the AM mostly unchanged. Even though secular disk heating processes also have an effect on heating newly formed stars, this effect is significantly lower than that induced by minor mergers, as already shown in Fig. 3.

\subsection{Rotational lag}

As the slowing down is only affecting the old stellar population, we expect to see an increasing difference between the AM content of the old and new stellar components as mergers proceed. This is indeed the case, as shown in Figs. 9 and 10, where the distribution of the specific AM, $l$, and radial and tangential velocities ( $v_{\mathrm{r}}$ and $v_{\mathrm{t}}$, respectively) of stars between radii of 2 and $3 R_{\mathrm{d}}$ are shown after a single minor merger and after two consecutive mergers. We chose this radial region as it should be representative of the solar neighborhood. To compare the effects of consecutive mergers to those of secular evolution, we show in Fig. 11 the same distributions for three galaxy simulations that do not undergo a merger but are evolved in isolation: for $\mathrm{gSa}$ and gSb model galaxies, and for a gas-rich, unstable clumpy disk. In this plot we show the newly formed stars as well as the entire old star population and the contribution of old stars at heights $|z| \leq 1 \mathrm{kpc}$, in order to distinguish old stars distributed in a thin disk from the total. We emphasize that this is a very simple way to distinguish between a thin, dynamically cold stellar component and a thick and hotter stellar component, which is based on the analysis of the vertical properties of thick disks formed in minor mergers (Qu et al. 2011).

The results of this study show that:

- after a single merger the thin stellar disk (i.e., at $|z| \leq 1 \mathrm{kpc}$ ) consists of two different stellar components, a young and an old population, the latter with a lower angular momentum than the former. In the region between 2 and $3 R_{\mathrm{d}}$, for a gS$\mathrm{bdSb}$ direct merger, where both galaxies initially have a gas fraction of $20 \%$, the old stars in the thin disk have an average specific AM which is about $10 \%$ lower than the new stars;

- if the whole old stellar population is considered, its average specific AM is about $7 \%$ lower than that of old stars at $|z| \leq 1 \mathrm{kpc}$, indicating that the stars in the thick old disk $(|z| \geq 1 \mathrm{kpc})$ contribute to decrease the specific AM and are thus less rotationally supported than the old stars in the thin disk;

- this difference in specific AM content of the stellar populations is reflected in different tangential velocities of their stars in the disk, with the tangential support of stellar orbits decreasing from the thin new disk, through the thin old disk, to the thick old disk;

- for the same gas fraction in the progenitor disks there is a difference between direct and retrograde mergers, in the sense that the specific AM content of old stars is lower if the satellite orbit is retrograde. This lower AM content can be explained by the presence of counter-rotating material (negative $l$ ) which is found in all retrograde encounters analyzed (see Sect. 3.4 for a detailed discussion);

- if one defines the rotational lag of old stars as $v_{\text {lag }}=v_{*}-$ $v_{\text {newstars}}$, where $v_{*}$ is the tangential velocity of stars in the thin or thick old disk, and $v_{\text {newstars }}$ is that of new stars, one can see that it depends on the amount of gas present in the progenitor disks: for old thin and old thick disk stars, the higher the gas fraction in the progenitor disk, the lower the rotational lag in the remnant.

We refer the reader to Table 3 for a complete summary of the results found in Figs. 9-11.

The difference between the specific AM content of old and new stars becomes still more pronounced after a second, consecutive minor merger, leading to a further increase in the rotational lag of both the old thin and thick disk stars, as shown in Fig. 10. Given that minor mergers have a cumulative impact on heating and slowing down stellar disks (see also Fig. 7), two mergers of mass ratio 1:10 are expected to result in a difference between the specific AM content of old and new stars that is comparable with that of one merger of mass ratio 1:5. In other words, more massive satellites can have a larger impact on slowing down the stellar disk and produce a larger rotational lag between the old and new stellar populations than less massive satellites (Qu et al. 2010). The substantial difference between the specific AM content of the thin and thick disk stellar populations cannot be due to secular evolution processes alone, which produce a considerably smaller variation in both $l$ and $v_{\mathrm{t}}$ in $3 \mathrm{Gyr}$ of evolution (Fig. 11). Note also that secular evolution produces a narrower distribution of $l, v_{\mathrm{r}}$ and $v_{\mathrm{t}}$, thus suggesting that also mixing and radial migration due to secular processes may be less effective than in minor mergers. Whereas scattering of stars by massive clumps can induce a rotational lag (in old thin and thick disk stars), the overall impact of scattering by mass concentrations is (moderately) lower than that produced by a direct 1:10 merger on a galaxy with an initial gas fraction of $20 \%$.

\subsection{Accreted and heated disk stars}

\subsubsection{Contribution to the rotational lag}

In the previous section we saw that old stars in the thin and thick disk of a minor merger remnant lag with respect to the new stars, and that this lag is higher for stars further from the galaxy midplane $(|z| \geq 1 \mathrm{kpc})$. But what is this rotational lag due to - is it mostly associated to stars originally in the satellite or in the primary disk, and how does it depend on the orbital parameters?

To answer these questions, we analyzed the tangential velocity $v_{\mathrm{t}}$ of old stars at radii between 2 and $3 R_{\mathrm{d}}$ in four different regions above the disk midplane: $|z| \leq 1 \mathrm{kpc}, 1<|z| \leq 3 \mathrm{kpc}$, $3<|z| \leq 5 \mathrm{kpc}$ and $5<|z| \leq 10 \mathrm{kpc}$. Results from some representative mergers are shown in Fig. 12, where for each encounter we distinguished the primary stars from those originally in the satellite. This figure shows some interesting trends. First of all, there is a clear difference between direct and retrograde mergers. While in both cases the average $v_{\mathrm{t}}$ of old stars is lower than that of new stars (Table 3), the behavior of $v_{\mathrm{t}}$ as a function of height $z$ is significantly different. Direct mergers produce disks with a tangential velocity $v_{\mathrm{t}}$ which decreases with height, meaning that the rotational lag increases with $z$, whereas in retrograde mergers $v_{\mathrm{t}}$ is constant with height up to $z \sim 5 \mathrm{kpc}$, and decreases only at greater heights. The satellite stars, which in each region constitute only a small percentage of the total stellar content, 
Y. Qu et al.: Minor mergers and their impact on the kinematics of old and young stellar populations in disk galaxies

Table 3. Kinematics of stars in the post-merger thin and thick old thick disks and of the new stars in disk galaxies heated by different physical processes.

\begin{tabular}{|c|c|c|c|c|c|c|c|c|c|}
\hline \multirow[b]{2}{*}{ Heating mechanism } & \multirow[b]{2}{*}{$f_{\text {gas }}$} & \multicolumn{3}{|c|}{ Old stars $|z| \leq 1 \mathrm{kpc}$} & \multicolumn{3}{|c|}{ Old stars $|z|>1 \mathrm{kpc}$} & \multicolumn{2}{|c|}{ New stars } \\
\hline & & $\begin{array}{c}\text { Mass } \\
\text { [stellar mass] }\end{array}$ & $\begin{array}{c}v_{\mathrm{t}} \\
{\left[\mathrm{km} \mathrm{s}^{-1}\right]}\end{array}$ & $\begin{array}{c}\mathrm{Lag} \\
{\left[\mathrm{km} \mathrm{s}^{-1}\right]}\end{array}$ & $\begin{array}{l}\text { Mass fraction } \\
\text { [stellar mass] }\end{array}$ & $\begin{array}{c}v_{\mathrm{t}} \\
{\left[\mathrm{km} \mathrm{s}^{-1}\right]}\end{array}$ & $\begin{array}{c}\mathrm{Lag} \\
{\left[\mathrm{km} \mathrm{s}^{-1}\right]}\end{array}$ & $\begin{array}{l}\text { Mass fraction } \\
\text { [stellar mass] }\end{array}$ & $\begin{array}{c}v_{\mathrm{t}} \\
{\left[\mathrm{km} \mathrm{s}^{-1}\right]}\end{array}$ \\
\hline 1:10 merger (direct) & 0. & 0.38 & 214. & n.d. & 0.62 & 181. & n.d. & - & - \\
\hline $1: 10$ merger (retrograde) & 0. & 0.28 & 203. & n.d. & 0.72 & 176. & n.d. & - & - \\
\hline $1: 10$ merger $($ direct $)$ & 0.1 & 0.34 & 208. & 42. & 0.65 & 175. & 75. & 0.01 & 250. \\
\hline $1: 10$ merger (retrograde) & 0.1 & 0.23 & 190. & 60. & 0.77 & 179. & 71. & 0.001 & 250. \\
\hline 1:10 merger (direct) & 0.2 & 0.4 & 195. & 24. & 0.58 & 170. & 49. & 0.015 & 219. \\
\hline $1: 10$ merger (retrograde) & 0.2 & 0.24 & 183. & 41. & 0.75 & 172. & 52. & 0.004 & 224. \\
\hline $2 \times(1: 10)$ merger & 0.1 & 0.22 & 182. & 68. & 0.77 & 155. & 95. & 0.01 & 250. \\
\hline $2 \times(1: 10)$ merger & 0.2 & 0.25 & 162. & 52. & 0.74 & 144. & 70. & 0.01 & 214. \\
\hline secular & 0.1 & 0.79 & 230. & 24. & 0.19 & 210. & 44. & 0.015 & 254. \\
\hline secular & 0.2 & 0.91 & 214. & 16. & 0.07 & 200. & 30. & 0.019 & 230. \\
\hline clumpy & 0.5 & 0.53 & 203. & 17. & 0.49 & 183. & 37. & 0.016 & 220. \\
\hline
\end{tabular}

Notes. For old stars in the thin and the thick disks the rotational lag is defined with respect to the tangential velocity $v_{\mathrm{t}}$ of the new stars.

do not contribute significantly to the lag. Moreover, if one compares the tangential velocities of satellite and primary stars for direct orbits, one can find a variety of behaviors in the regions analyzed: in some cases the tangential velocities of satellite stars are always smaller than those of stars from the primary (e.g., for orbit "gS0dS001dir33"), while in other cases the values are comparable, and satellite stars show even higher velocities than primary stars in the outer regions (e.g. orbit "gSadSa01dir33"). In all the cases, however, the strongest variations in $v_{\mathrm{t}}$ as a function of $z$ are associated with stars originally in the primary rather than from the satellite. For retrograde orbits, the tangential velocities of satellite stars show only small variations with increasing $z$, as is the case for primary stars. We note also that a stellar thick disk formed in an unstable clumpy galaxy is characterized by tangential velocities whose variation with $z$ is very similar to that produced in a direct encounter with $f_{\text {gas }}=0.2$ (compare, e.g. Fig. 13 with orbit "gSbdSb01dir33" in Fig. 12).

\subsubsection{How to distinguish satellite stars}

Villalobos \& Helmi (2009) recently proposed an interesting method to use the vertical AM to discriminate between stars formed in the primary thin disk and then heated by a minor merger from those originally in the satellite and then accreted. Their simulations showed (see their Fig. 6) that satellite stars in the height zone $|z| \leq 1 \mathrm{kpc}$ have a typical AM content which is constant with radius, while stars from the primary disk tend to have an AM $l \propto r$. Their simulations are gas-free and involve satellites more massive than the ones studied in this paper. Because of these differences we decided to reinvestigate the validity of their method, exploring if the result is similar for larger mass ratios like ours (1:10) and for other satellite and primary galaxy morphologies. Our results for the specific AM of stars originally in the primary disk and in the satellite galaxy are shown in Figs. 14 and 15. We divided the stars into two regions, one at $|z| \leq 1 \mathrm{kpc}$ and another at $|z| \geq 1 \mathrm{kpc}$. The general result confirms that the difference between the specific AM of satellite stars and primary disk stars is significant in the outer disk regions: typically the difference is $20 \%$ at radii of $1.5-2 R_{\mathrm{d}}$. But at these radii the fraction of satellite stars with $|z| \leq 1 \mathrm{kpc}$ is only a few percent of the total number of stars. This means that it can be extremely difficult to detect these stars in an observational sample. We find, however, that the fraction of satellite stars is five to ten times larger at greater heights: at $|z| \geq 1 \mathrm{kpc}$, outside a radius of $1.5-2 R_{\mathrm{d}}$ the specific AM content of accreted stars can be significantly different from that of primary stars, and their fraction sufficiently higher (around 10\%) to make the observational detection of this signature more likely. Note however that generally the satellite stars with the lowest AM content are found very far from the galaxy midplane $(|z| \geq 5 \mathrm{kpc})$. We want to point out that there are also cases in which there is no discernible difference in the specific AM of satellite and primary disk stars at any radius, even at the largest radii plotted in Figs. 14 and 15). This is in agreement with what was found in Fig. 12, where primary and satellite stars show remarkably similar tangential velocities.

Obviously, the specific AM becomes a better discriminant if satellite orbits counter-rotate with respect to the primary disk - a clear sign of an external origin of stars. We find counterrotation in all merger remnants which formed from satellites on retrograde orbits (some examples are shown in Fig. 15). We will discuss the main properties of counter-rotating stars further in the next section.

\subsection{Counter-rotation}

Counter-rotating stellar disks are observed in external galaxies (see Yoachim \& Dalcanton 2005) and counter-rotation is also found in the Milky Way halo (see Carollo et al. 2007). The presence of stars (in the thick disk or in the halo) rotating in a direction which is reverse of the galaxy main spin cannot be explained with secular evolution processes, and is a strong evidence for an external origin.

It is thus of great interest to investigate the characteristics of counter-rotating stars in our merger remnants - specifically, their fraction and vertical distribution with radius. Examples are shown in Figs. 16 and 17 for a number of dissipationless and dissipative retrograde mergers. In these plots four different regions are selected: (1) at $|z| \leq 1 \mathrm{kpc} ;$ (2) at $1 \leq|z| \leq 3 \mathrm{kpc}$; (3) at $3 \leq|z| \leq 5 \mathrm{kpc}$; and (4) at $5 \leq|z| \leq 10 \mathrm{kpc}$. It is clear that counter-rotating stars are found at all values of $z$, from the galaxy midplane to $10 \mathrm{kpc}$ above it, and that the probability to find counter-rotating stars increases with height: the fraction $N_{\text {ct }} / N_{\text {tot }}$ of counter-rotating stars to the total number of stars in the region is $\leq 5 \%$ up to $|z| \leq 3 \mathrm{kpc}$, typically around $10 \%$ in the $3 \mathrm{kpc} \leq|z| \leq 5 \mathrm{kpc}$ and it can reach $30 \%$ in the region highest above the disk. This trend is also due to the fact that the fraction of satellite stars $N_{\mathrm{s}}$ to the total number of stars increases with $z$. In other words, it is the overall increase in the fraction of satellite 
stars with $z$ which determines this trend, not simply a decrease in the number of disk stars as a function of height above the disk. Furthermore the fraction of counter-rotating stars depends also on the initial orbital inclination of the satellite: for a satellite on an orbit inclined by 60 degrees with respect to the primary disk, this fraction is lower at all $z$ (Villalobos \& Helmi 2008).

If counter-rotating stars are a natural outcome of low orbital inclination in single minor mergers with satellites on retrograde orbits, would a second retrograde merger then increase the fraction of counter-rotating stars even further at all heights? In our models (Fig. 18) the effect of a second retrograde merger with the same mass ratio is to increase the fraction of counter-rotating stars by a factor of about two, at all heights. We can conclude from this that repeated retrograde minor mergers have a cumulative effect on the number of counter-rotating stars in the thick disk and inner halo.

\subsection{Kinematics of the remnant disk}

The AM evolution of the baryonic component during merging processes discussed in the Sect. 3.1 is naturally reflected in an evolution of the line-of-sight velocity $v_{\mathrm{los}}$ and the $v_{\mathrm{los}} / \sigma_{\mathrm{v}}$ ratio. Minor mergers always produce a decrease in $v_{\text {los }}$ and an increase in the velocity dispersion $\sigma_{\mathrm{v}}$ of the stellar component at all radii (Fig. 19). The remnant stellar disks usually have a smaller $v_{\mathrm{los}} / \sigma_{\mathrm{v}}$ ratio than the initial values before interaction. Consecutive satellite accretions have cumulative effects on decreasing the $v_{\mathrm{los}}$ and increasing the $\sigma_{\mathrm{v}}$, and thus deceasing the $v_{\mathrm{los}} / \sigma_{\mathrm{v}}$ ratio. Nevertheless, smaller variations in both $v_{\mathrm{los}}$ and $\sigma_{\mathrm{v}}$ can be found in minor mergers with gas, compared to gasfree merger cases, due to the fact that dissipative gas can help preserve disk rotation by forming new rotating disk stars during mergers. The decreases in $v_{\text {los }}$ and the $v_{\text {los }} / \sigma_{\mathrm{v}}$ ratio indicate that stellar disks become hotter and rotate more slowly after minor merger events, which is consistent with the AM loss and the increase of the anisotropy parameter $\beta$ (Figs. 4 and 7).

\section{Discussion and conclusions}

By means of $N$-body/SPH simulations, we studied the kinematics of stars in galaxy remnants of 1:10 mass ratio minor mergers. The simulated interactions span a range in gas mass fractions, from 0 to 0.2 in the primary and satellite galaxies, and a range of orbital parameters.

As shown in Qu et al. (2010), minor mergers result in a redistribution of orbital into internal angular momentum, which affects all galaxy components. In particular, old stars, i.e. those already in place before the interaction, always lose angular momentum during the merging process. The decrease of the specific AM of old stars is accompanied by a redistribution of stellar orbits, as traced by the anisotropy parameter $\beta$, which become increasingly radial. In minor mergers with gas in the disk of the primary galaxy we find a similar trend of old stars losing angular momentum and as a result their orbits becoming more radially dominated. However, when a new stellar component forms from gas present in the primary disk during the merger, its AM content is significantly different: the orbits tend to be more tangentially dominated, thus providing a higher rotational support. This different behavior results in a final stellar disk with two different stellar populations with significantly different AM content. In particular, old stars always show a rotational lag with respect to the young stellar component. If one separates all stars into thin disk stars (at heights $|z| \leq 1 \mathrm{kpc}$ from the galaxy midplane) and thick disk stars (at $|z|>1 \mathrm{kpc}$ ), three different components can be found, with different dynamical properties: (1) young stars in the thin disk, which are rotationally supported and show the highest values of $v_{\mathrm{t}} ;(2)$ old thin disk stars lagging with respect to the new stars; and (3) old thick disk stars lagging with respect to both thin disk components. For a minor 1:10 merger, with a satellite accreted on a direct orbit and with an initial primary disk gas fraction of 0.2 , the old stars in the thin disk have a rotational lag of about $20 \mathrm{~km} \mathrm{~s}^{-1}$, while the old stars in the thick disk have a velocity about $50 \mathrm{~km} \mathrm{~s}^{-1}$ lower than the young stellar component, both lag values being compatible with the estimates for the Milky Way (see Gilmore et al. 2002). Multiple mergers can further reduce the tangential velocity of the old stellar components, while leaving that of the new stars mostly unchanged, thus resulting in a further increase in rotational lag with every successive accretion episode. As the two populations, old stars and new stars, have different tangential velocities, in a plot of $v_{\mathrm{t}}$ as function of age we expect to find a discontinuity at the time when the merger occurs. Of course, the newly formed stars will evolve in time, and if no other merger takes place they will be slowly heated by secular effects. However, as we have shown in Fig. 3, secular processes are much less effective in altering stellar kinematics than minor mergers. Therefore, in our opinion, a discontinuity in the age- $v_{\mathrm{t}}$ plane (or the age- $\beta$ parameter) between the old and new stellar populations should still be visible, even if secular processes, and asymmetric drift in particular, contribute to the slow heating of the new stellar populations. Unfortunately, the hybrid particle method we adopted to implement star formation in the simulations does not allow us to follow properly the heating of the newly formed stars with time. We do think, however, that this discontinuity in the age- $v_{\mathrm{t}}$ behavior may be a distinctive feature of minor mergers compared to secular processes in galaxy disks, which deserves to be modeled accurately in the near future.

Minor mergers can quantitatively reproduce the increase in the rotational lag with height above and below the galaxy midplane that was found for the Milky Way (Chiba \& Beers 2000; Girard et al. 2006), but only if the satellite is accreted along a direct orbit; for retrograde orbits no trend of the rotational lag with $z$ is found, up to distances of $5 \mathrm{kpc}$ from the midplane, above which it even decreases slightly. Together with the increasing trend of stellar eccentricities with height (Di Matteo et al. 2010), this is another piece of evidence suggesting that if the Milky Way thick disk has formed through heating of a pre-existing thin disk by minor merger(s), the orbit of the satellite(s) should have been prograde.

Recently, Bournaud et al. (2009) proposed a scenario in which stellar thick disks could have formed in gas-rich galaxies at high redshifts, through scattering of stars by massive clumps. Comparing the kinematics of minor merger remnants with those of thick disks formed in clumpy disks we find that, under certain conditions, both scenarios can reproduce a rotational lag of the old thin and old thick disk stars that are compatible with observations for the Milky Way. In particular, disks heated by massive clumps show a rotational lag which increases with height $z$, as found for minor mergers on direct orbits. Even so, the two processes leave distinctly different signatures in the vertical surface profiles of the remnant disks, as shown in Qu et al. (2011). Obviously, disks heated by massive clumps cannot lead to the counter-rotating thick disks observed in external galaxies (Yoachim \& Dalcanton 2008). For this to occur, an external accretion of material (gas or stars) is necessary.

Counter-rotating material is found in all our simulations where the satellite is accreted along a retrograde orbit. The amount of counter-rotating stars (all associated to stars initially 
Y. Qu et al.: Minor mergers and their impact on the kinematics of old and young stellar populations in disk galaxies

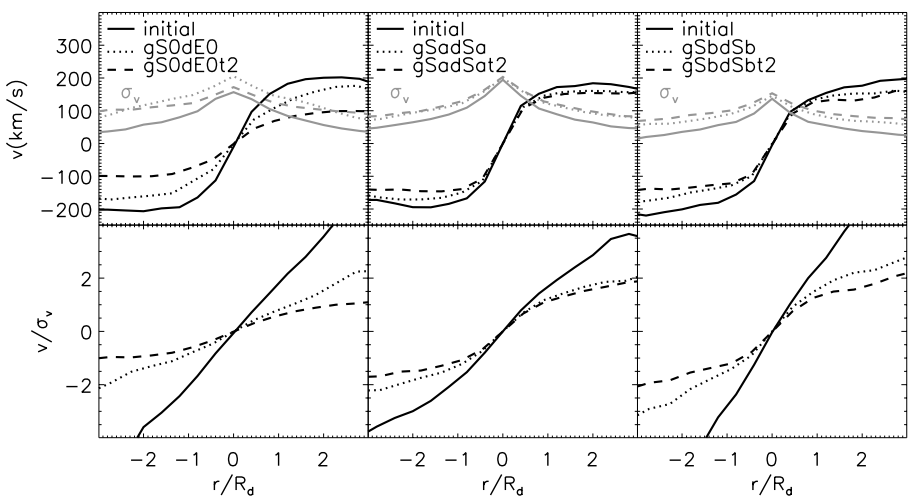

Fig. 19. Line-of-sight velocity, $v$, velocity dispersion, $\sigma_{\mathrm{v}}$, and the $v / \sigma_{\mathrm{v}}$ ratio as a function of scaled radius in remnant stellar disks after both a single and two consecutive dissipative minor mergers. The same data are also shown for the initial stellar disks, before merging. The gas mass fraction of the merging galaxies increases from the left to the right, from 0 for the $\mathrm{S} 0$ case, to 0.2 for the Sbs.

in the satellite galaxy) increases with $z$ as a result of the increasing contribution of satellite stars to the whole stellar content, and at $z=3-5 \mathrm{kpc}$ the fraction of counter-rotating stars is between $10 \%$ and $15 \%$. We showed that the effect of multiple mergers on the generation of a counter-rotating disk is cumulative, in the sense that the fraction of counter-rotating stars doubles after the accretion of a second, comparatively massive, satellite on a retrograde orbit. It is thus possible that some of the galaxies observed to have a high fraction of counter-rotating material in the thick disk (Yoachim \& Dalcanton 2005) could have accreted a number of satellites on retrograde orbits. The fraction of counter-rotating stars we find in our minor merger simulations is quantitatively consistent with the amount of counterrotation observed in the thick disks of nearby galaxies (Yoachim \& Dalcanton 2005).

Finally, we discussed the possibility of discriminating stars accreted from the satellite galaxy from those formed in the primary disk on the basis of their AM content, as recently proposed by Villalobos \& Helmi (2009). We find that in general the AM content is a better discriminant for stars located at vertical distances from the galaxy midplane greater than $1 \mathrm{kpc}$ rather than at smaller heights, as proposed by Villalobos \& Helmi (2009). Moreover, as the fraction of satellite stars increases with $z$ in our models, their detection at high $z$ should also be more likely. However, we want to point out that it may not always be possible to use the AM content to distinguish between stars originally in the primary and those accreted from the satellite because there are cases where no difference in their AM content is seen at any radius.

Overall, the qualitative and some quantitative agreement with observations of the thick disk of the MW and other nearby galaxies suggest that minor merger remains a viable mechanism for forming the thick disk. With future observations, it may be possible to search for other signs of the impact of minor mergers on disk galaxies, such as determining the relative angular momentum of stars as a function of age in nearby disk galaxies.
Minor merger models, such as those discussed here, make specific predictions which can be tested observationally (e.g., by GAIA).

Acknowledgements. Y.Q. and P.D.M. are supported by a grant from the French Agence Nationale de la Recherche (ANR). We are grateful to Benoît Semelin and Françoise Combes for developing the code used in this paper and for their permission to use it. These simulations will be made available as part of the GalMer simulation data base (http://galmer.obspm. fr). We wish to thank Gary Mamon for his extensive and useful comments and the anonymous referee for a careful, in-depth and constructive report which helped improve the paper considerably.

\section{References}

Athanassoula, E. 2005, NYASA, 1045, 168

Barnes, J. E. 1992, ApJ, 393, 484

Bendo, G. J., \& Barnes, J. E. 2000, MNRAS, 316, 315

Bird, J. C., Kazantzidis, S., \& Weinberg, D. H. 2011, MNRAS, accepted [arXiv: 1104.0933B]

Bournaud, F., Combes, F., Jog, C. J., \& Puerari, I. 2005a, A\&A, 438, 507

Bournaud, F., Combes, F., \& Semelin, B. 2005b, MNRAS, 364, 18

Bournaud, F., Jog, C. J., \& Combes, F. 2005c, A\&A, 437, 69

Bournaud, F., Elmegreen, B. G., \& Martig, M. 2009, ApJ, 707, L1

Bournaud, F., Chapon, D., Delaye, L., et al. 2011, ApJ, 730, 4

Carollo, D., Beers, T. C., Lee, Y. S., et al. 2007, Nature, 450, 1020

Chiba, M., \& Beers, T. C. 2000, AJ, 119, 2843

Chilingarian, I., Di Matteo, P., Combes, F., Melchior, A.-L., \& Semelin, B. 2010, A\&A, 518, 61

Crocker, A. F., Jeong, H., Komugi, S., et al. 2009, MNRAS, 393, 1255

Debattista, V. P., Mayer, L., Carollo, C. M., et al. 2006, ApJ, 645, 209

Di Matteo, P., Bournaud, F., Martig, M., et al. 2008a, A\&A, 492, 31

Di Matteo, P., Combes, F., Melchior, A.-L., \& Semelin, B. 2008b, A\&A, 477, 437

Di Matteo, P., Jog, C. J., Lehnert, M. D., Combes, F., \& Semelin, B. 2009, A\&A, 501,9

Di Matteo, P., Lehnert, M. D., Qu, Y., \& van Driel, W. 2010, A\&A, 525, L3

Fakhouri, O., \& Ma, C.-P. 2008, MNRAS, 386, 577

Gilmore, G., Wyse, R. F. G., \& Norris, J. E. 2002, ApJ, 574, 39

Girard, T. M., Korchagin, V. I., Casetti-Dinescu, D. I., et al. 2006, AJ, 132, 1768

Hopkins, P. F., Cox, T. J., Younger, J. D., \& Hernquist, L. 2009, ApJ, 691, 1168

Ibata, R., Irwin, M., Lewis, G., Ferguson, A. M. N., \& Tanvir, N. 2001, Nature

Jesseit, R., Cappellari, M., Naab, T., Emsellem, E., \& Burkert, A. 2009, MNRAS, 397, 1202

Jog, C. J., \& Maybhate, A. 2006, MNRAS, 370, 891

Klement, R. J. 2010, A\&ARv, 18, 567

Mapelli, M., Moore, B., \& Bland-Hawthorn, J. 2008, MNRAS, 388, 697

Martinez-Delgado, D., Gabany, R. J., Crawford, K., et al. 2010, AJ, 140, 962

McConnachie, A. W., Irwin, M. J., Ibata, R. A., et al. 2009, Nature, 461, 66

Mihos, J. C., \& Hernquist, L. 1994, ApJ, 427, 112

Minchev, I., Famaey, B., Combes, F., et al. 2011, A\&A, 527, 147

Naab, T., \& Burkert, A. 2003, ApJ, 597, 893

Puerari, I., \& Pfenniger, D. 2001, Ap\&SS, 276, 909

Qu, Y., Di Matteo, P., Lehnert, M., van Driel, W., \& Jog, C. J. 2010, A\&A, 515, A11

Qu, Y., Di Matteo, P., Lehnert, M., \& van Driel, W. 2011, A\&A, 530, A10

Quillen, A. C., Minchev, I., Bland-Hawthorn, J., \& Haywood, M. 2009, MNRAS, 397, 1599

Reichard, T. A., Heckman, T. M., Rudnick, G., et al. 2009, ApJ, 691, 1005

Semelin, B., \& Combes, F. 2002, A\&A, 388, 826

Toomre, A. 1977, Evolution of Galaxies and Stellar Populations, Proceedings of a Conference at Yale University, ed. B. M. Tinsley, \& R. B. Larson (New Haven: Yale University Observatory)

Villalobos, A., \& Helmi, A. 2008, MNRAS, 391, 1806

Villalobos, A., \& Helmi, A. 2009, MNRAS, 399, 166

Yoachim, P., \& Dalcanton, J. 2005, ApJ, 624, 701

Yoachim, P., \& Dalcanton, J. J. 2008, ApJ, 682, 1004 

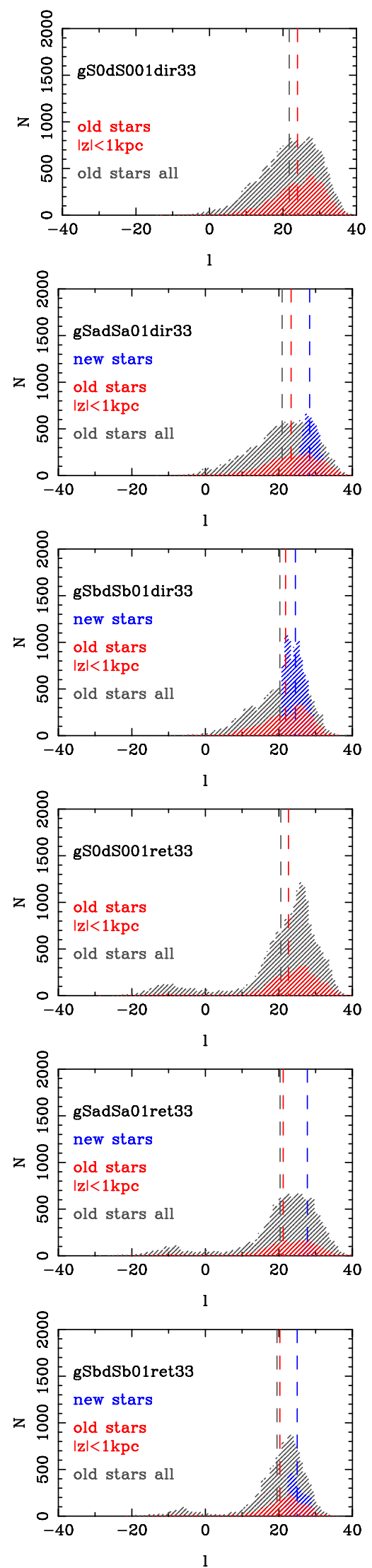
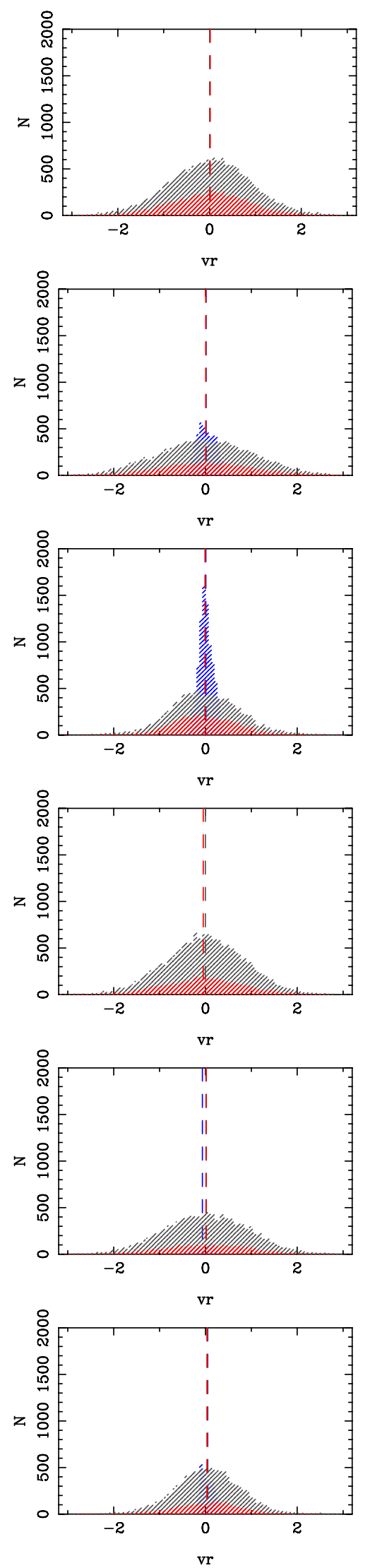
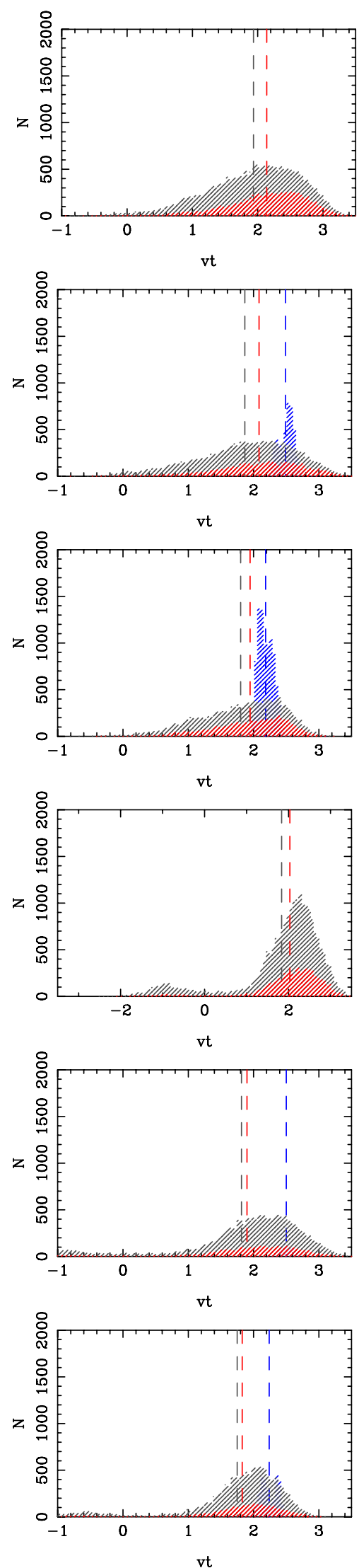

Fig. 9. Histograms of the specific AM, $l$ (in units of $100 \mathrm{kpc} \mathrm{km} \mathrm{s}^{-1}$ ), and the radial $\left(v_{\mathrm{r}}\right)$ and tangential $\left(v_{\mathrm{t}}\right)$ velocities (in units of $\left.100 \mathrm{~km} \mathrm{~s}^{-1}\right)$ of stars between radii of $2-3 R_{\mathrm{d}}$ in a number of minor merger remnants. The top three rows show direct minor mergers, with disk gas mass fractions increasing from 0 to 0.2 . Indicated in each panel are the entire old stellar population (gray), new stars (blue) and old stars with $|z| \leq 1 \mathrm{kpc}$ (red). The vertical dashed lines show the average values of $l, v_{\mathrm{r}}$ and $v_{\mathrm{t}}$ for each of these three components. The three lowest rows are similar, but for retrograde mergers. 
Y. Qu et al.: Minor mergers and their impact on the kinematics of old and young stellar populations in disk galaxies
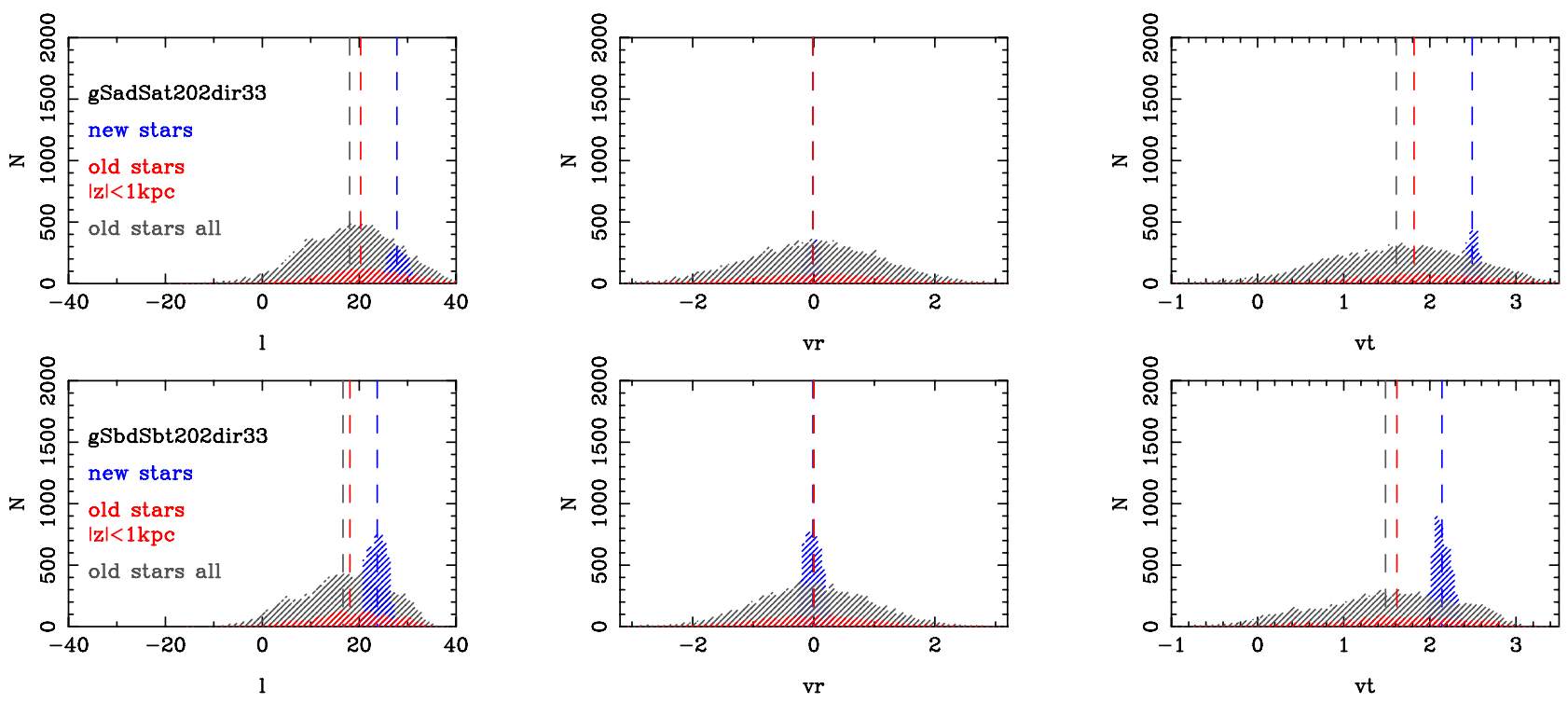

Fig. 10. Same as Fig. 9, but for two consecutive minor mergers.
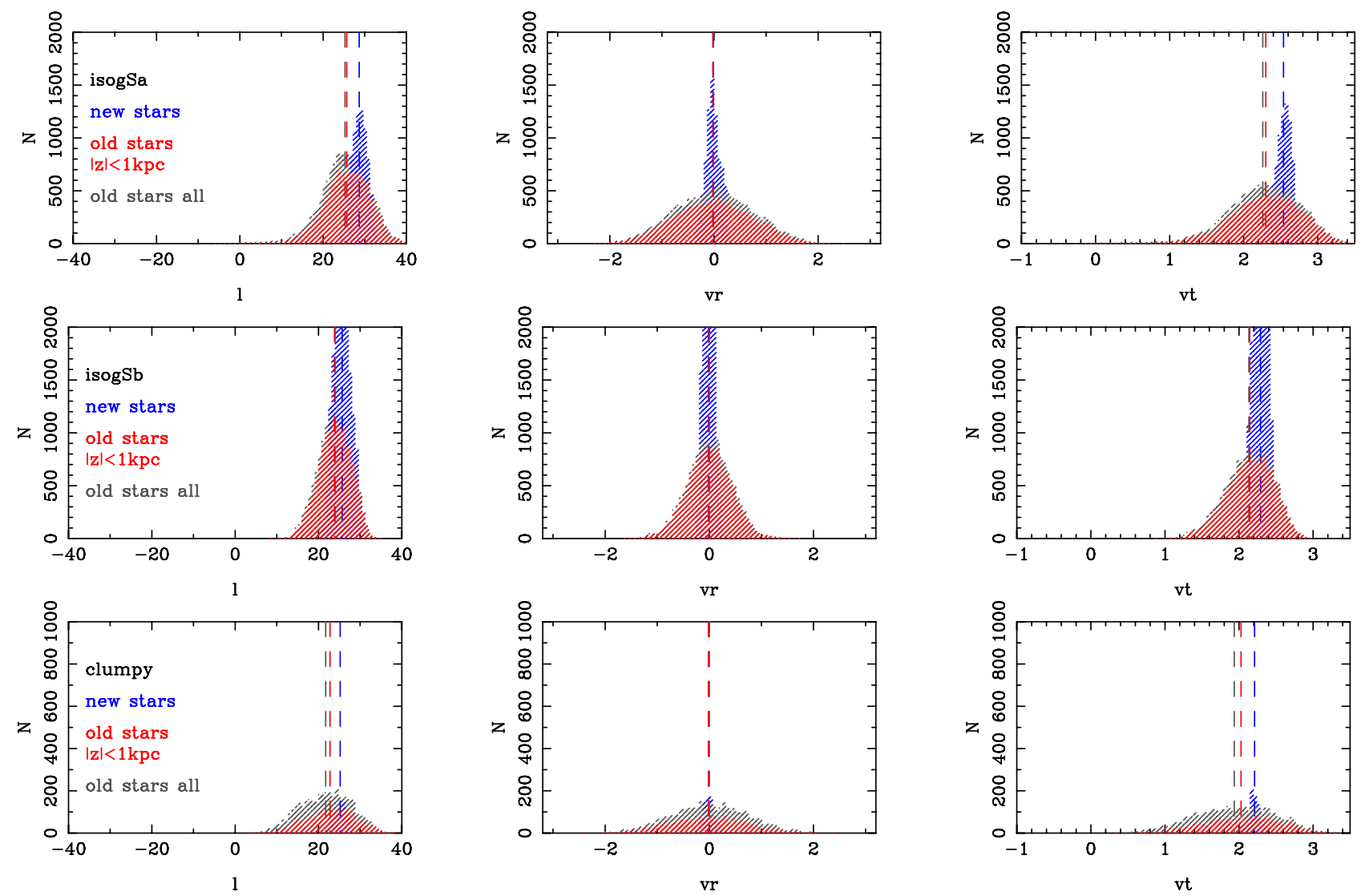

Fig. 11. Same as Fig. 9, but for galaxies that were evolved in isolation for 3 Gyr: a gSa (upper panels) with a gas mass fraction $f_{\text {gas }}=0.1$ and a $\mathrm{gSb}$ (middle panels) with $f_{\text {gas }}=0.2$, as well as a galaxy with a much higher gas fraction of 0.5 which went through an unstable clumpy phase (bottom panels). In the latter case the parameters are also shown after $3 \mathrm{Gyr}$, when the clumps have dissolved through tidal effects or spiraled into the galaxy center through dynamical friction and interaction. 

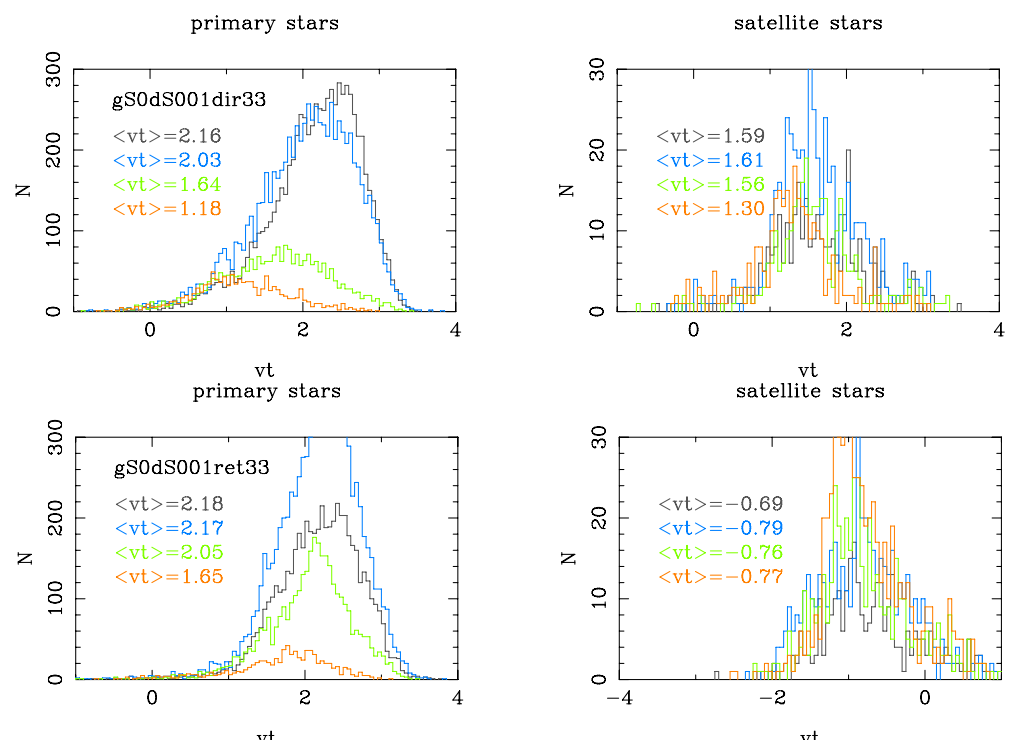

primary stars

satellite stars
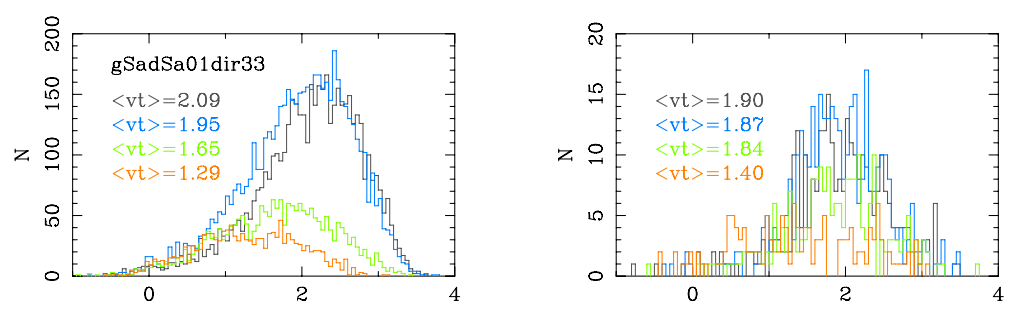

primary stars

satellite stars

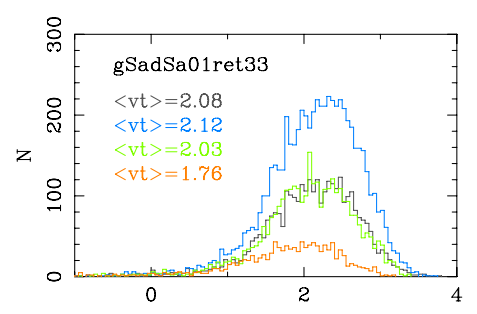

vt

primary stars

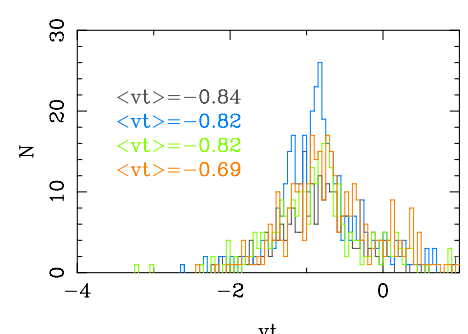

satellite stars

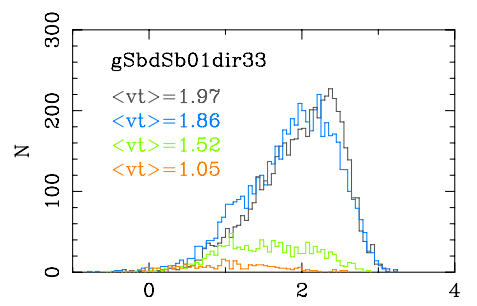

vt

primary stars

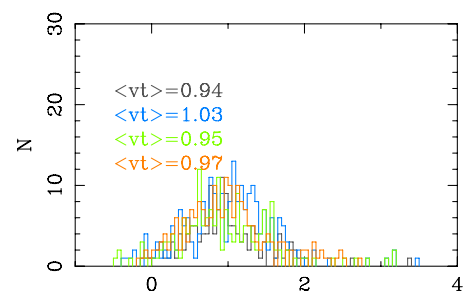

satellite stars

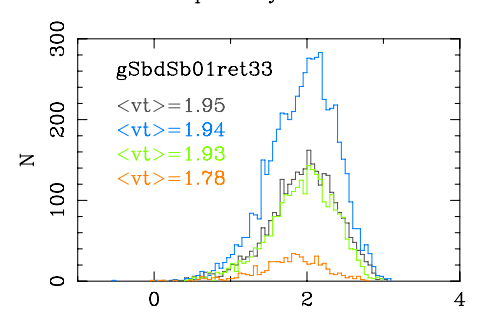

vt

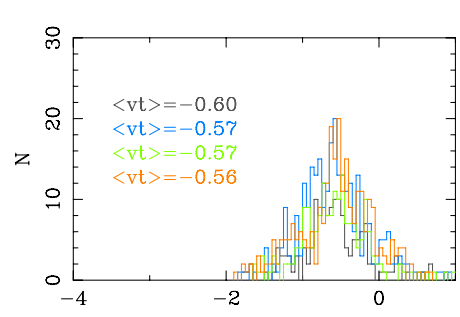

vt

Fig. 12. Distribution of tangential velocities $v_{\mathrm{t}}$ (in units of $100 \mathrm{~km} \mathrm{~s}^{-1}$ ) of old stars for six minor mergers. For each encounter a pair of panels is shown, with stars originally in the primary disk (left) and those accreted from the satellite (right). In each panel the histograms correspond to different heights above and below the galaxy midplane: $|z| \leq 1 \mathrm{kpc}$ (black), $1<|z| \leq 3 \mathrm{kpc}$ (blue), $3<|z| \leq 5 \mathrm{kpc}$ (green) and $5<|z| \leq 10 \mathrm{kpc}$ (orange). The average values of $v_{\mathrm{t}}$ in the different regions is also indicated. 
Y. Qu et al.: Minor mergers and their impact on the kinematics of old and young stellar populations in disk galaxies

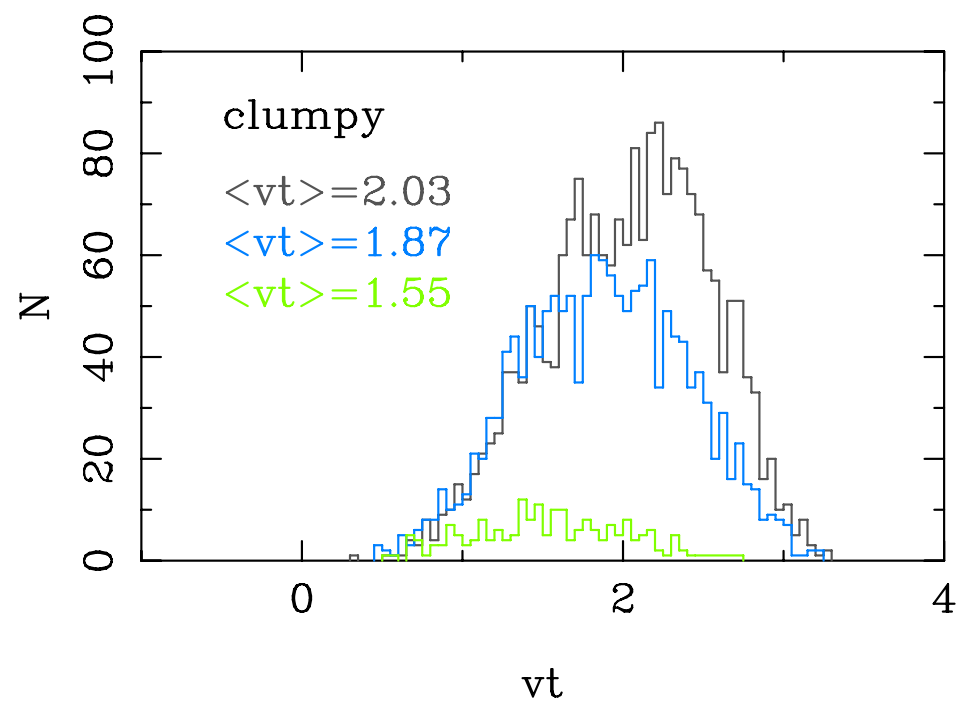

Fig. 13. Same as Fig. 12 but for an isolated, very gas-rich galaxy $\left(f_{\text {gas }}=0.5\right)$, after an unstable, clumpy phase. Note that in this case the fourth and highest region is missing, due to a lack of stars above/below $5 \mathrm{kpc}$ from the galaxy midplane. 
A\&A 535, A5 (2011)
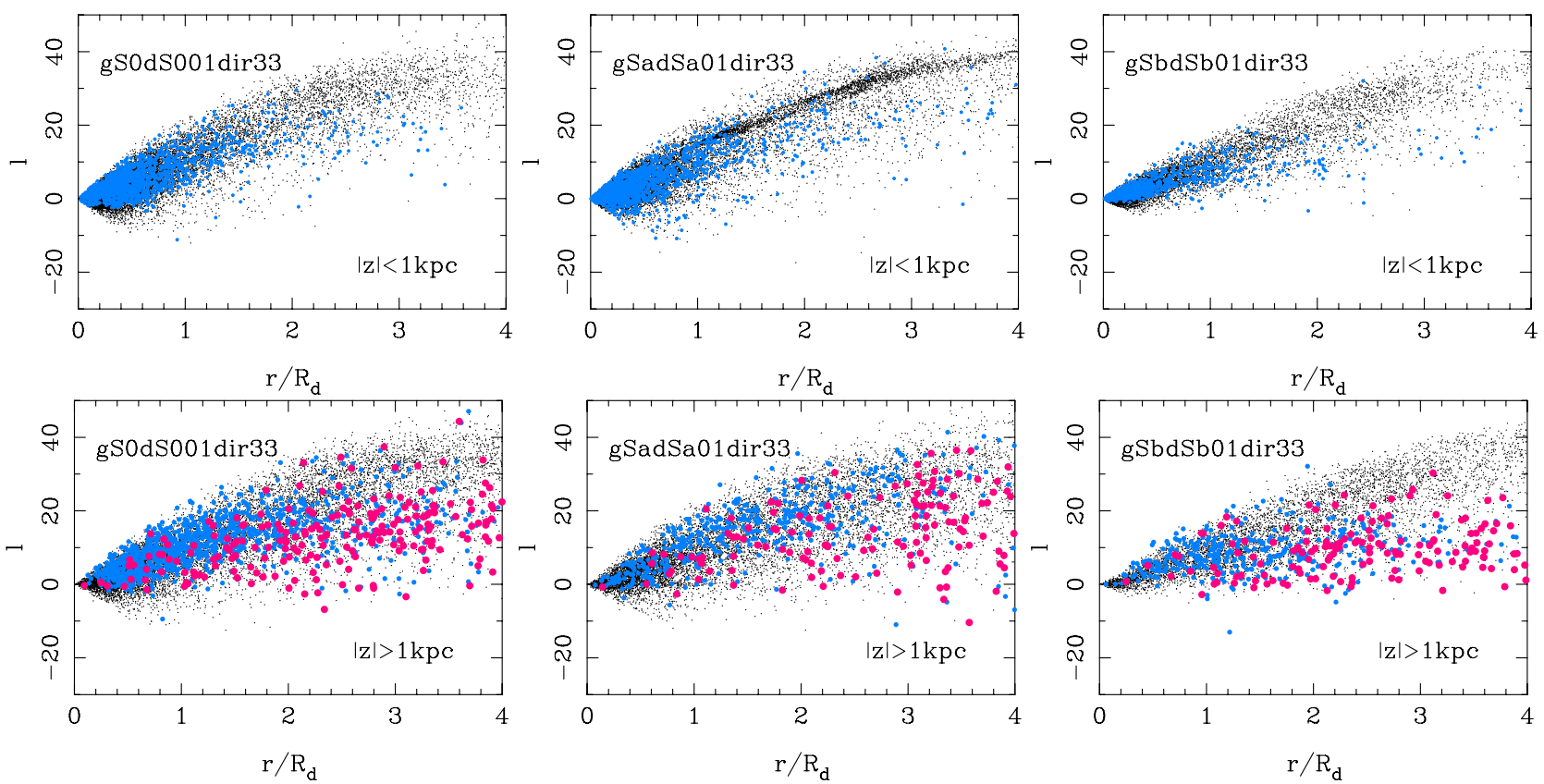

Fig. 14. Specific AM, $l$, as a function of scaled radius for old stars in three direct merger remnants. For each encounter a pair of plots is shown, where stars are selected on their heights above (or below) the galaxy midplane: $|z| \leq 1 \mathrm{kpc}$ (upper panels) and $|z|>1 \mathrm{kpc}$ (lower panels). In each panel stars originally in the primary galaxy are shown as black points, stars originally in the satellite as blue points, and satellite stars with $|z|>5 \mathrm{kpc}$ as red points. The specific AM is in units of $100 \mathrm{kpc} \mathrm{km} \mathrm{s}^{-1}$.
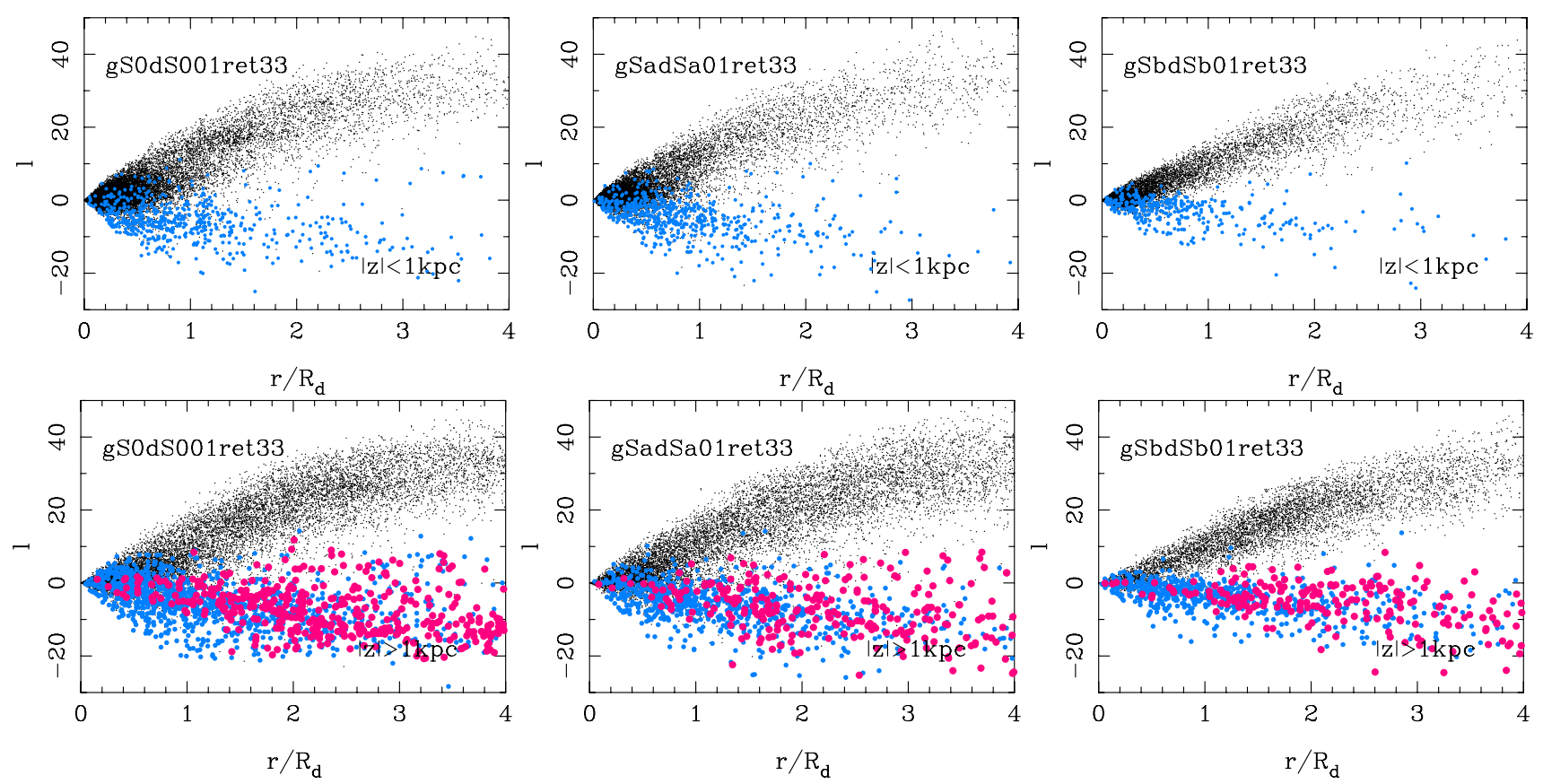

Fig. 15. Same as Fig. 14, but for three retrograde mergers. 

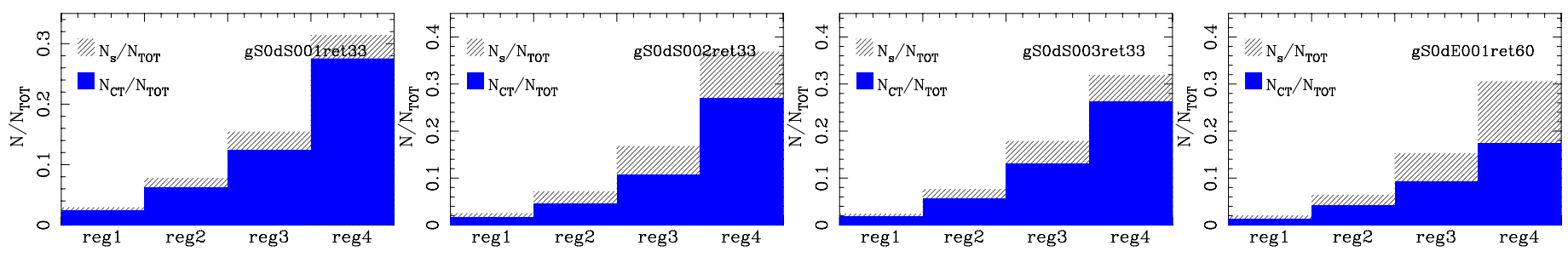

Fig. 16. Fraction of counter-rotating stars (shown in blue) for four dissipationless retrograde mergers. Stars are divided into four regions, according to their height above (or below) the galaxy midplane: (1) at $|z| \leq 1 \mathrm{kpc}$; (2) at $1 \mathrm{kpc} \leq|z| \leq 3 \mathrm{kpc}$; (3) at $3 \mathrm{kpc} \leq|z| \leq 5 \mathrm{kpc}$; and (4) at $5 \mathrm{kpc} \leq|z| \leq$ $10 \mathrm{kpc}$. For each region the fraction of satellite stars to the total number of stars is also shown (gray).
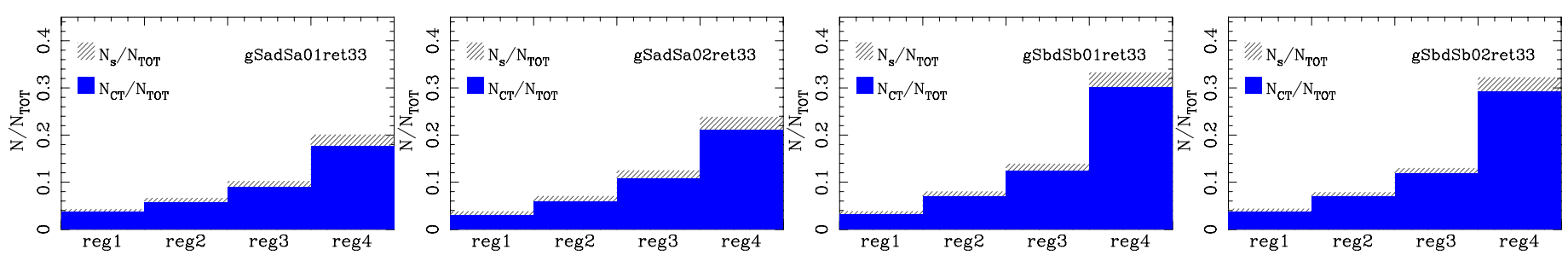

Fig. 17. Same as Fig. 16, but for four dissipative retrograde mergers.
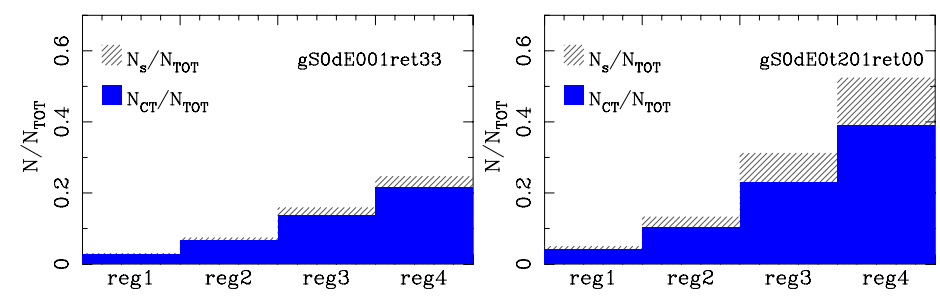

Fig. 18. Same as Fig. 16, but for a gS0 galaxy after a single retrograde merger (left panel) and after two consecutive retrograde mergers (right panel) with a dE0 satellite. 